
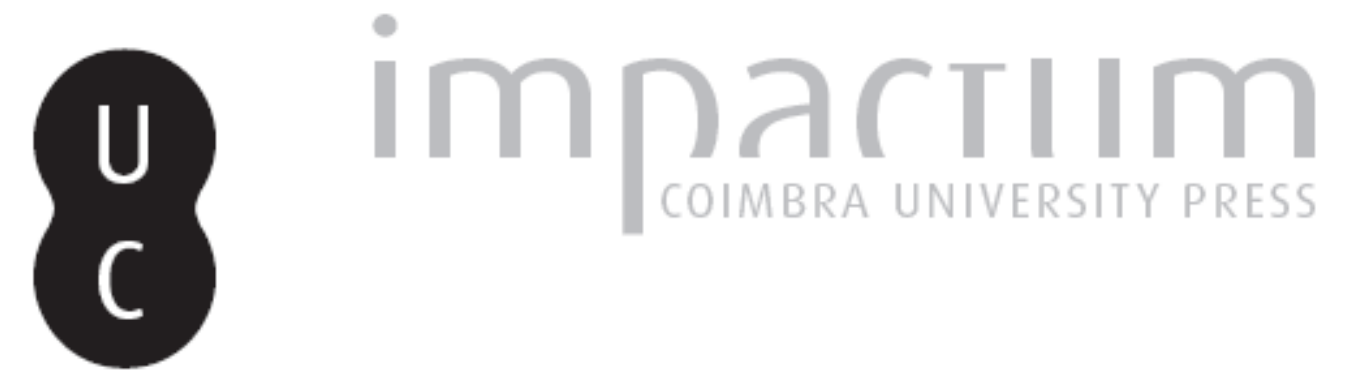

\title{
La mosaïque d'Océan découverte à Faro: (Algarve)
}

\section{Autor(es): Lancha, J.}

Publicado por: Imprensa da Universidade de Coimbra

URL persistente:

URI:http://hdl.handle.net/10316.2/45658

DOI:

DOI:https://dx.doi.org/10.14195/1647-8657_24_7

Accessed : $\quad$ 26-Apr-2023 09:35:53

A navegação consulta e descarregamento dos títulos inseridos nas Bibliotecas Digitais UC Digitalis, UC Pombalina e UC Impactum, pressupõem a aceitação plena e sem reservas dos Termos e Condições de Uso destas Bibliotecas Digitais, disponíveis em https://digitalis.uc.pt/pt-pt/termos.

Conforme exposto nos referidos Termos e Condições de Uso, o descarregamento de títulos de acesso restrito requer uma licença válida de autorização devendo o utilizador aceder ao(s) documento(s) a partir de um endereço de IP da instituição detentora da supramencionada licença.

Ao utilizador é apenas permitido o descarregamento para uso pessoal, pelo que o emprego do(s) título(s) descarregado(s) para outro fim, designadamente comercial, carece de autorização do respetivo autor ou editor da obra.

Na medida em que todas as obras da UC Digitalis se encontram protegidas pelo Código do Direito de Autor e Direitos Conexos e demais legislação aplicável, toda a cópia, parcial ou total, deste documento, nos casos em que é legalmente admitida, deverá conter ou fazer-se acompanhar por este aviso.

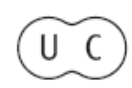


FACULDADE DE LETRAS

INSTITUTO DE ARQUEOLOGIA

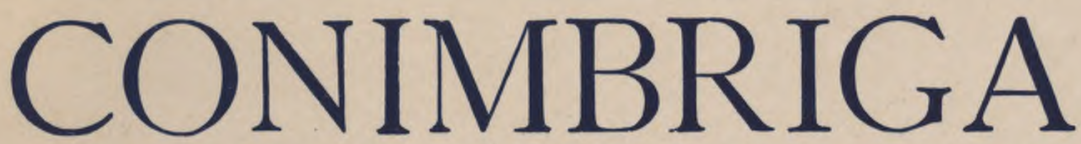

VOLUMEXXIV

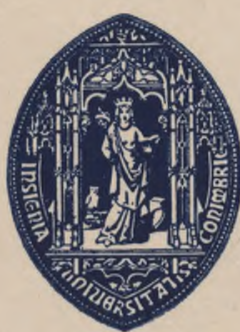

UNIVERSIDADE DE COIMBRA

1985 
J. LANCHA

Membre scientifique de la Casa de Velâzquez (Madrid)

\author{
LA MOSAÏQUE D'OCÉAN DÉCOUVERTE À FARO (ALGARVE) \\ «Conimbriga» XXIV (1985), p. 151-175
}

RÉSU MÉ : La mosaïque découverte en 1976, déposée par Madame A. Alarcâo et M. C. Beloto, et actuellement au Musée de Faro, n'avait fait l'objet jusqu'ici que d'une brève présentation. L'A. consacre une étude détaillée à ce pavement, d'une importance archéologique exceptionnelle puisqu'il constitue le premier véritable témoin de l'urbanisme d'Ossonoba. Le décor végétal du tapis, et l'iconographie des Vents et du masque d'Océan du tableau central font l'objet de comparaisons avec des exemples de la Péninsule Ibérique et de l'ensemble $\mathrm{du}$ monde romain. L'A. établit une relation entre l'origine africaine du type du masque d'Océan et son exécution à Faro par un atelier africain ou inspiré des modèles de ces ateliers. L'inscription qui accompagne le pavement donne les noms des quatre commanditaires du pavement; l'A. propose une nouvelle restitution de la lacune à la 3 . ème ligne et montre que cette mosaïque ornait un édifice public dent les évergètes — quattuor-viri du municipe ou membres d'un collège religieux ou professionnel — ont utilisé la mosaïque comme support pour immortaliser leur geste civique: phénomène assez peu courant à l'époque de la mosaïque, c.à.d. la fin du II ème ou le début du III ème siècle.

Resumo: O mosaico descoberto em 1976, consolidado por Adília Alarcão e Carlos Beloto, actualmcnte exposto no Museu de Faro, só fora objecto de uma pequena nota de apresentação.

A Autora estuda agora, em pormenor, este pavimento cuja importância arqueológica é excepcional, uma vez que constitui o primeiro testemunho real do urbanismo de Ossónoba. A decoração vegetal e a iconografia dos Ventos e do rosto do Oceano são comparados com outros exemplos da Península Ibérica e do mundo romano; a Autora aponta, nomeadamente, a possibilidade de a origem africana do tipo de máscara do Oceano ser um índice de 
que o mosaico foi executado em Faro por urna oficina africana ou inspirada em modelos africanos.

A Autora propõe uma nova reconstituição para a lacuna da linha 3 da inscrição e mostra que o mosaico pertencia a um edificio público, cujos evergetas - quattuor-viri do municipio ou membros de um colégio religioso ou profissional - quiseram imortalizar, assim, o seu gesto cívico: circunstância não muito frequente na época a que o mosaico se reporta, ou seja, os finais do séc. $\mathrm{n}$ ou princípios do $\mathrm{m}$. 


\section{LA MOSAÏQUE D'OCÉAN DÉCOUVERTE À FARO (ALGARVE) *}

Elle fut aperçue pour la première fois le 27 Avril 1976, à l'angle des rues de l'Infant Dom Henrique et Ventura Coelho à Faro (fig. 1). Complètement dégagée en 1979, elle fut alors déposée par l'équipe du Musée monographique de Conimbriga et installée, après transfert sur un nouveau support, dans une salle du musée archéologique municipal Infante Dom Henrique à Faro. Nous voudrions présenter quelques réflexions d'ordre archéologique et iconographique qui permettent de situer le pavement dans son contexte ibérique, après la brève présentation de la découverte et de la restauration due à A. Alarcão, C. Beloto, J. d'Encarnaçào et M. M. de Almeida C).

Deux photographies partielles prises à la découverte $\left({ }^{2}\right)$ et un dessin d'ensemble (3) levé par les restaurateurs permettent de repérer les lacunes dans ce pavement de 9,40 m X 3,40 m. Elles sont dues à l'installation de tuyauteries modernes $\left({ }^{4}\right)$. N'ayant pu

* C'est pour nous un agréable devoir de remercier chaleureusement Madame A. Alarcão pour son aide généreuse dans la préparation de cet article, et M. Pinheiro e Rosa, Directeur du musée archéologique municipal Infante Dom Henrique à Faro, pour son autorisation d'étudier ce pavement.

0) Cf. A. Alarcĩo, C. Beloto, J. D'encarnaçao, M. M. de almeida, O mosaico do Oceano de Faro, in «Anais do Município de Faro», X, 1980, p. 219-232. Les auteurs y exposent la méthode de dépose et d'installation de la mosaïque au Musée de Faro, et donnent une première étude iconographique et épigraphique de la mosaïque.

${ }^{(2)}$ ID., ibid., fig. 2.

$\left.{ }^{3}\right)$ ID., ibid., fig. 1.

(4) La pose d'une tuyauterie fournit précisément l'occasion de la découverte, en 1976.

Conimbriga, 24 (1985), 151-175 
réaliser une vue d'ensemble de la mosaïque exposée au Musée, nous reproduisons le dessin cité supra (fig. 2).

La mosaïque était assez bien conservée. Les lacunes se réduisent à deux rangées d'hexagones dans la partie supérieure, au tiers inférieur du tableau central, et à une partie de l'inscription et de la bordure extérieure du pavement. Elles ne nuisent pas à la cohérence de l'ensemble. La mosaïque ornait une pièce environ trois fois plus longue que large, qui évoque une entrée de grandes dimensions.

D'autre part, la dépose et la restauration ont été exemplaires. Seules les lacunes ponctuelles ont été comblées avec des tesselles de récupération dans les parties géométriques et dans la tête d'Océan. Mais dans ce dernier cas, il est facile de se faire une idée de leur faible ampleur en comparant le revers de la tête du dieu, débarrassée de son mortier antique $\left({ }^{5}\right)$ et la vue prise à la découverte $\left(^{6}\right)$ avec la photographie de la tête telle qu'on peut la voir aujourd'hui au Musée (fig. 3).

La ville d'Ossonoba que l'on s'accorde à situer à Faro (Algarve) était déjà attestée par des inscriptions encastrées dans la muraille de la ville, et par la découverte d'un temple dans le Largo da Sé, ainsi que d'une nécropole $\left({ }^{7}\right)$.

Des fragments de mosaïque avaient déjà été mentionnés dans les fouilles du Largo da Sé, en 1933 et en 1941 ( $^{8}$ ) mais aucune mosaïque complète n'était apparue dans la partie de la ville qui recouvre la ville romaine. La mosaïque d'Océan est donc la première des mosaïques de Faro que l'on connaisse. Par ailleurs, une monnaie autonome, frappée à l'avers d'un bateau et au revers de deux poissons, résume les activités principales de la ville, située sur

${ }^{(5)}$ ID., ibid., fig. 3 .

(6) ID., ibid., fig. p. 221.

(7) Voir sur ce point l'état de la question présenté par M. L. Estício da Veiga Affonso dos Santos dans son livre Arqueologia romana do Algarve, Lisbonne, 1971, vol. I, p. 166 sqq. et par J. Alarcão, Portugal romano, Lisbonne, 1973, p. 80 sqq., 157, 175 et 187 . En relation avec la nécropole a été également découvert un important groupe de lampes, publiées par G. Lyster Franco, Lucernas romanas, $\mathrm{m}$ «Actas e Memórias do I Congresso Nacional de Arqueologia», Lisbonne, 1970, p. 161-187, 16 pi.

(8) Cf. A. Vian a, Restos de Ossonoba, in «Revista do Sindicato Nacional dos Engenheiros...», n. ${ }^{\circ}$ 39-46, 1949, p. 1-36. 
un estuaire, et toute proche de la mer. On a également découvert à Faro une usine de salaisons $\left({ }^{9}\right)$. La mosaïque d'Océan qui, comme nous le verrons infra, ornait un édifice public de la ville, s'insère donc parfaitement par son sujet dans le contexte d'un port sur l'Atlantique. Rappelons au passage qu'un temple aux dieux des eaux a été découvert dans la ville voisine de Milreu $\left({ }^{10}\right)$.

Les données de fouille sont bien peu nombreuses, et difficiles à utiliser, étant donné qu'il s'agit d'une fouille de sauvetage, menée par des conservatrices du Musée archéologique de Lisbonne, et qui n'a pas été publiée. Nous devons à l'amabilité de Madame A. Alarcão les quelques informations qui vont suivre, au sujet $\mathrm{du}$ contexte archéologique du pavement. Elles sont le fruit des observations faites sur place par Madame A. Alarcão.

La mosaïque était recouverte d'une couche de terre de $3 \mathrm{~m}$ d'épaisseur, due à l'alluvionnement. Une fouille fut effectuée sous le niveau du pavement. On distinguait:

1 - le niveau de pose des tesselles.

2 - le nucleus du lit de pose.

3 - le rudus du lit de pose.

4 - une couche de cendres mêlées d'abondants matériaux de destruction (fragments de stucs, de peinture murale, de brique et de tuiles).

5 - un sol d'argile blanche mêlée de sable et de chaux.

6 - une couche de terre argileuse et de cailloux qui semblait être le lit de pose du précédent.

7 - une couche très épaisse de cendres.

La mosaïque d'Océan appartient donc au dernier état de l'édifice, dans un secteur de la ville marqué par deux niveaux de destruction successifs, dus à des incendies. Il faut signaler des fragments de mosaïque de couleur noire, blanche et rouge, exposés dans la vitrine du Musée de Faro qui est consacrée au matériel découvert pendant cette fouille de sauvetage. Nous supposons

$\left({ }^{9}\right)$ In M. L. Estácio da Veiga, o. I., vol I, p. 186.

(10) Mentionné dans J. Alarcĩo, o. p. 80 et étudié par Th. Hauschild, dans son article MilreujEstoi (Algarve) «M. M.», 21, 1980, p. 189-219. 
qu'ils appartenaient au pavement de la couche 5, dont le nucléus (argile blanche mêlée de sable et de chaux) était en place. Il y a donc eu au moins un pavement antérieur à la mosaïque d'Océan, à moins que ces fragments ne proviennent d'une pièce voisine. La céramique découverte sous la couche 5 remontait au 1er s. après J.C., selon Madame A. Alarcão ( $\left.{ }^{n}\right)$. Dans la salle où est exposée la mosaïque sont également présentés six fragments de peinture murale découverts au même endroit; il s'agit d'imitations de marbres sur fond ocre, à plinthe bleue.

\section{La mosaïque actuelle (fig. 2)}

Elle présente un tapis rectangulaire de $9,40 \times 3,40 \mathrm{~m}$, avec une première bordure extérieure sur le petit côté correspondant à l'entrée. Cette bordure à rinceau noir, à simples volutes, n'a que deux volutes de conservées. Sur les quatre côtés, une bordure de triangles noirs sur fond blanc, entre deux filets noirs, de $0,16 \mathrm{~m}$ de large, borde le tapis, délimité lui-même par un filet noir, de 1,5 cm de large. Le tapis est divisé en quatre panneaux:

un tableau géométrique de $2,45 \times 2,95 \mathrm{~m}$ (deux fois)

un tableau central figuré de $2,45 \mathrm{~m}$ de côté

un tableau avec inscription, de 0,42 de large X 2,45 m (anses comprises).

Le panneau avec inscription, se présente comme une tabula ansata et les lettres sont écrites en capitales noires sur fond blanc, sur trois lignes.

Deux panneaux géométriques à décor de dodécagones sécants encadrent le tableau central carré où l'on voit, inscrit dans un cercle, une tête d'Océan. Le cercle est entouré de deux bordures, l'une, une tresse à deux brins sur fond noir, l'autre, composite, comprend successivement une ligne brisée de fuseaux (Rép. graphique n. ${ }^{\circ}$ 177) et une ligne de peltes noires sur fond blanc. Les

(") D'après les notes des conservatrices du Musée Archéologique de Lisbonne, des tessons de TSC1 A se trouvaient dans la couche 2, ce qui nous donne un terminus post quem pour la mosaïque d'Océan du début du 2ème s. 
fuseaux et les peltes ont un remplissage de tesselles de couleur ocre.

Dans les écoinçons de la partie supérieure, deux têtes de Vents. Les deux autres, dans la partie inférieure du carré, étaient détruites à la découverte, ainsi que le tiers inférieur du disque.

Sur le dessin dû à A. Alarcâo et C. Beloto, le pavement est complété dans sa partie supérieure (deux rangées d'hexagones), à juste titre nous semble-t-il, car la composition est bien évidemment symétrique de part et d'autre du tableau central.

Par commodité, nous décrirons successivement le décor végétal, le décor du tableau central et l'inscription.

Le décor végétal des hexagones - qui en inscrivent chacun un second, dessiné par un filet ocre, rose, noir, est varié: 27 types de fleurons, dont deux seulement sont repris deux fois. Dans les carrés sur la pointe, un même fleuron est repris dans tout le pavement (un quatre-feuilles lancéolées noires, timbrées d'une croisette blanche); dans les carrés droits, un noeud de Salomon gris bleu et ocre, avec une croisette blanche en son centre.

\section{Le répertoire végétal (fig. 4 et $4 \mathrm{a}$ )}

De haut en bas et de gauche à droite:

I, a, b, c: perdus

II, a, b, c: perdus

III, a: incomplètement conservé: 4 fleurs de lotus sur tige, marron à nervure blanche, autour d'un bouton central circulaire blanc, inscrivant un second cercle noir, avec une tesselle noire centrale. Couleurs: ocre, rose, gris, noir. Deux intervalles symétriques sont reliés par une pelte noire.

b: quatre-feuilles lancéolées (ocre) autour d'un bouton central circulaire dessiné par un filet noir et orné d'une ligne de trois tesselles ocre sur la pointe. Des arabesques (un filet noir) sertissent les feuilles et sont reliées entre elles par une accolade ocre.

IV, a: quatre-feuilles lancéolées (noir, gris) autour d'un bouton central circulaire dessiné par un filet noir et timbré d'une fleurette noire, et quatre-feuilles bilobées (ocre, rose) sur tige.

b : deux peltes grises symétriques dessinées par un filet noir reliées par leurs extrémités, et deux feuilles bilobées (ocre, rose) 
sur tige, autour d'un bouton central circulaire dessiné par un fdet noir, avec une tesselle noire en son centre.

c: quatre-feuilles trifides (ocre, rose, noir) et quatre palmettes à trois branches autour d'un bouton circulaire dessiné par un fdet noir et timbré d'une fleurette noire.

V, a: quatre-feuilles trifides (ocre, rose, noir) et quatre-feuilles lancéolées (marron, noir) autour d'un bouton central circulaire dessiné par un filet noir avec une tesselle noire en son centre.

b: quatre-feuilles découpées (ocre, rose) autour d'un bouton central circulaire dessiné par un filet noir et timbré d'une fleurette noire.

VI, a: quatre-feuilles trifides (ocre, rose) et quatre-feuilles lancéolées tournoyantes (gris, noir) autour d'un bouton central circulaire dessiné par un filet noir et timbré d'une fleurette noire.

b: quatre-feuilles lancéolées (ocre, rose) et quatre tiges surmontées de postes opposées dessinées par un fdet noir, avec une tesselle noire sur la pointe entre les deux peltes.

c: quatre-feuilles lancéolées (gris, noir) et quatre-feuilles cordiformes (ocre, rose) la pointe tournée vers le centre, sur tige aboutissant au bouton central circulaire dessiné par un filet noir et timbré d'une fleurette noire.

VII, a: trois-feuilles lancéolées (gris, noir) et trois-feuilles identiques (ocre, rose) autour d'une tesselle noire centrale.

$\mathrm{b}$ : quatre-feuilles trifides (ocre, rose, marron, noir) et quatre-feuilles lancéolées (marron, rose) autour d'un bouton central circulaire dessiné par un fdet noir et timbré d'une fleurette.

Vili,a: - VII,a

$$
\mathrm{b}:=\mathrm{VI}, \mathrm{b}
$$

IX, a: quatre-feuilles trifides (ocre, marron, noir) et quatrefeuilles lancéolées symétriques à l'extrémité d'une tige noire, autour d'un bouton circulaire dessiné par un fdet noir et timbré d'une fleurette rose, ocre, noire.

b: = III,a, sans pelte noire dans les intervalles, remplacée par un petit triangle à base dentelée,

c: - VI,c.

$\mathrm{X}$, a: proche de VII,b, Vili,b, XI,a,b, et XIII,a.

b: quatre-feuilles bilobées (ocre, rose) reliées par des arabesques noires au bouton central. 
$\mathrm{XI}$, a et $\mathrm{b}:=\mathrm{X}, \mathrm{a}, \mathrm{b}$.

c: proche de IV,b, avec une feuille cordiforme et deux postes opposées symétriques par rapport à la pointe de la pelte.

XII, a: quatre-feuilles lancéolées noires alternant avec quatre palmettes simples (marron, gris, noir) autour d'un bouton circulaire dessiné par un filet noir avec une tesselle noire en son centre.

b: $-\mathrm{X}$

XIII, a: proche de IX,b et III,a.

b iquatre-feuilles trifides (rose, ocre, noir) alternent avec quatre-feuilles ayant la forme de glands (marron, noir), montées sur une tige dont les tesselles sont posées sur la pointe. Bouton central dessiné par un filet à fond rose et timbré d'un petit carré de tesselles blanches dont les angles sont reliés au bord du cercle.

c: deux feuilles lancéolées alternent avec deux feuilles de lotus noires, de forme très allongée; ces dernières sont surmontées d'une tesselle noire. Au centre, une rosette de tesselles blanches.

XIV, a: quatre-feuilles trifides (marron, noir) alternent avec quatre-feuilles lancéolées en fuseau (marron, rose) avec bouton central circulaire, dessiné par un filet noir et timbré d'une croisette noire.

$\mathrm{b}$ : huit peltes sur tige, croisées en diagonale, affrontées deux à deux (4 roses, 4 noires) avec au centre quatre petits triangles à base dentelée, la pointe tournée vers le croisement des tiges.

En dépit de leur assez grande variété (27 types différents, sans tenir compte des variantes d'un même type) tous ces fleurons appartiennent à un répertoire d'ambition modeste. Aucun fleuron n'est composite, et cela s'explique en partie par les contraintes de l'espace disponible dans un hexagone aux côtés irréguliers $(0,20$ et $0,30 \mathrm{~m}$ de côté). La palette de couleurs est réduite à quatre, qui ne sont pas choisies pour créer une illusion de type naturaliste, mais sans doute en fonction des disponibilités du mosaïste. Si l'on excepte le rose, toutes ces couleurs font partie du répertoire de base courant. 
On relève un certain schématisme dans l'exécution $\left({ }^{12}\right)$; il atteint quelquefois les limites de la stylisation, assez réussie en XIII,c. Si deux d'entre eux sont repris de part et d'autre du tableau central, tous les autres constituent des créations originales qui sont autant de variantes à partir d'éléments de base communs. C'est le propre du travail d'un atelier qui emploie plusieurs mosaïstes à la fois pour exécuter la partie la moins importante d'un pavement commandé peut-être ici par les quattuor-viri A'Ossonoba.

D'autre part, un répertoire végétal étendu associé au thème océanique peut surprendre, nous aurons à nous demander infra s'il s'agit d'un choix conscient ou arbitraire du commanditaire.

Précisons enfin que nous n'avons pu faire de prélèvements de tesselles pour analyser les matériaux. Mais il semble que les couleurs employées dans la partie géométrique et dans les têtes des Vents soient des calcaires, probablement locaux. Il serait intéressant de déterminer l'origine du calcaire rose pâle qui revient fréquemment dans les fleurons et les encadrements. En revanche, dans la tête d'Océan dominent les marbres et les tesselles de verre. La palette est complètement différente, beaucoup plus chaude et variée.

Le tableau centrai

\section{Les Vents (flg. 5)}

En haut à gauche: buste d'un Vent personnifié qui regarde vers l'extérieur du tapis; quelques lacunes dans l'aile, le sourcil, l'oreille, la bouche et la tête. Imberbe et juvénile, il peut s'agir

(12) On observe un répertoire végétal beaucoup plus sec dans deux autres mosaïques de la région, à Amendoal (cf. M. L. Estácio da Veiga, $o . I$., vol. II, p. 174 et fig. 248, 250). On trouve un répertoire complètement différent à Conimbriga, qui constitue un véritable répertoire régional. On a ici une série de fleurons qui, par leur caractère un peu frêle et par leur coloris, évoquent plutôt certains fleurons africains, de Bulla Regia et Acholla. Dans une autre province de l'Empire, la Gaule, on note en revanche un véritable répertoire régional, facilement reconnaissable, à Vienne (cf. J. LANС н A, Florilège viennois, in «Mélanges H. Stern», Paris, 1983, p. 244-251). 
de Zéphyr ou d'Eurus (fig. 5 a). Traits principaux: mâchoire forte, visage rond, front bombé et menton accentué. Le contour du visage est dessiné en tesselles rose, grenat, rouge veiné, selon la direction de la lumière. Les zones claires du visage en tesselles beige, le reste en tesselles roses et grenat, de forme régulière; l'arcade sourcilière et la pupille sont noires, les cheveux roses (quatre tons), et certaines boucles sont soulignées de noir, ainsi que l'oreille, pour donner plus de relief à l'ensemble.

Il ne reste que quelques tesselles de l'aile, sur le haut du front (tesselles gris bleu). Les dégradés de couleur plus que le soin apporté à la direction des lignes de tesselles donnent à ce visage une certaine expressivité.

La densité des tesselles au $\mathrm{dm}^{2}$ est de $110(0,9 \mathrm{~cm}$ de côté pour chaque tesselle).

En haut à droite: buste d'un Vent qui regarde vers l'extérieur du tapis; quelques lacunes dans l'aile et les cheveux (fig. 5 b). Il est vu de profil, comme le précédent. En dépit du collier de barbe (grenat et noir) qui permet de l'identifier avec Boréas, le vent du Nord, la tête de ce dernier est exécutée avec plus de soin que la précédente.

Elle est empreinte d'une certaine gravité. La palette est la même; le nez et l'oeil sont mieux dessinés.

L'aile sur le front est bien conservée (tesselles de verre turquoise, bleu outremer, bleu canard) et les cheveux sont traités avec soin (nombreuses mèches de couleur grenat, rose, ocre, noir). On remarque l'usure des tesselles de verre. Même densité que dans le précédent.

\section{La tête d'Océan (fig. 6)}

Vue de trois-quarts à gauche, conservée sur presque toute sa hauteur $(0,60 \mathrm{~m})$. Le dieu regarde vers sa droite et lève les yeux au ciel. Quelques lacunes dans le menton, la moustache et la barbe. Les tesselles sont particulièrement usées dans la pince à gauche et dans les antennes inférieures.

Toutes les tesselles employées, à part un calcaire jaune dans les pinces, semblent être des tesselles de marbre, ou de verre. Elles ont de 0,5 à $1 \mathrm{~cm}$ de côté dans la tête d'Océan, et $0,5 \mathrm{~cm}$ 
dans le fond blanc crème, exécuté avec autant de soin que la figure elle-même. Les interstices vont de 1 à $2 \mathrm{~mm}$, l'exécution est soignée (densité de 150 tesselles au $\mathrm{dm}^{2}$ ).

S'il est probable que le même mosaïste n'a pas exécuté les bustes des Vents et la tête d'Océan, il est clair que c'est la même équipe qui a travaillé aux sujets figurés, si l'on en juge par la palette utilisée dans les deux cas. Toutefois, la gamme de couleurs est plus soutenue dans la tête d'Océan.

La tête se détache sur un fond clair et se caractérise par la recherche d'expressivité: traits accusés, visage émacié, long nez, sourcils froncés, bouche bien modelée, lumières et ombres distribuées sur le front, le nez, les joues. La technique utilisée pour la pose des tesselles, du front à la naissance des sourcils, est le réticulé.

La chevelure est également traitée en deux zones, zone sombre près du visage, zone plus claire au second plan (marron clair, rose). La même répartition de l'ombre et de la lumière s'observe dans le rendu des pinces et des antennes. Par ailleurs, le front bas contribue à donner à ce visage un air sombre, propre à un homme d'âge mûr, pas à un vieillard. Dans la moustache, la barbe et les dauphins - dont il ne reste qu'une partie de la nageoire dorsale et du dos -, la couleur verte évoque le milieu marin dans lequel évolue le dieu. Mais le mosaïste a apporté un soin tout particulier au rendu des yeux:

- oeil gauche rond, cerné de grenat, dessiné par deux lignes de tesselles orange, au sourcil noir et grenat. Le fond de l'oeil est blanc et gris bleu, avec un iris noir dessiné par trois tesselles rectangulaires cernées de grenat.

- oeil droit dont la ligne est plus vague, sans contour net; les couleurs sont disposées en dégradé, du clair au foncé.

Séparée de son contexte marin, la tête d'Océan est traitée ici comme un masque, d'après un carton donnant les lignes de force du visage. L'association des Vents avec une tête d'Océan traitée, comme ici, sous les traits d'un masque, n'est pas surprenante mais elle n'est pas la plus fréquente dans les représentations du dieu. Les Vents personnifient des forces naturelles $\left({ }^{13}\right)$. Sans

(1S) Dans les mosaïques d'Avenches I (Winde vielmustermosaik), in V. von Gonzen васн, Die römische Mosaiken der Schweiz, Bâle, 1961, pi. 71; 
doute, le schéma adopté pour le tableau central; un médaillon dans un carré - a-t-il influencé le mosaïste dans le choix des bustes des Vents en écoinçon. Océan figure ici sur un fond de couleur neutre.

Pour situer cette tête d'Océan dans son contexte ibérique, il apparaît que sur les dix exemples connus dans la Péninsule Ibérique $\left({ }^{14}\right)$ deux ou trois seulement associent cette image avec

de Frampton, in E. Rivet ed., The Roman Villa in Britain, Londres, 1969, pl. 3.28 (IVème s.); de Sousse (L. Foucher, Inventaire des mosaïques de Sousse, Tunis, 1960, n. 57 168); peut-être la mosaïque d'Amphitrite à Tagiura; et, dans la Péninsule Ibérique, les mosaïques de Balazote (in S. DE Los S Antos Gallego et R. Sanz Gamo, Museo de Albacete, Albacete, 1982, une planche en couleurs), de Mérida (mosaïque cosmologique), de Milla del Rio, et de Faro.

(14) Contre 21 dans les provinces d'Afrique. Avec ses 10 exemples, la Péninsule Ibérique se place en seconde position dans le monde romain par le nombre des découvertes. Si l'on dresse une liste récapitulative à partir de la dernière étude publiée sur ce sujet, par A. Paulian, Le dieu Océan en Espagne: un thème de Vart hispano-romain, «M.C.V.», 15, 1979, p. 115-133, on peut citer, en allant du nord au sud:

- Milla del Rio, $c f$. en dernier lieu, T. Mañanes., Astorga romana y su entorno, Valladolid, 1983, p. 144 et pi. XXVIII.

- Lugo (voir biblio. dans A. Paulian, art. cit.).

- Dueñas (Palencia), (voir biblio. dans A. Paulian, art. cit.).

- Quintanilla de la Cueza, $c f$., en dernier lieu, M. A. García Guinea, Guia de la villa romana de Quintanilla de la Cueza, Palencia, 1982, pièce n. ${ }^{\circ}$ 3, p. 26 sq. et une seconde mosaïque, de même sujet et dans un cadre géométrique différent, dans la pièce $n .^{\circ} 24$, fig. 41 (en couleurs).

- Balazote (v. biblio., note 13)

- Cordoue: n. ${ }^{\circ}$ 1: en dernier lieu dans le Corpus de mosaicos de España, fase. Ili, Cordoba, Jaén, Málaga, Madrid, 1981, n. ${ }^{\circ} 6$.

n. ${ }^{\circ}$ 2: mosaïque signalée dans le journal ABC, 29-10-1981, et présentée dans Arqueologia 81, Madrid, 1982, une vue de détail en couleurs, p. 152.

- El Chorreadero (Paterna) : in Corpus de mosaicos de España, fase. IV, Sevilla, Granada, Cádiz y Murcia, Madrid, 1982, n. ${ }^{\circ}$ 49, pl. 21. Selon notre identification, il s'agit d'une tête d'Océan, intégrée dans un cortège marin, dont il reste des Tritons et des poissons. La tête est fragmentaire, mais les éléments caractéristiques conservés en partie ne permettent pas le doute: la barbe mêlée d'algues, une antenne, une oreille en forme de queue de langouste. Elle est exécutée en noir et blanc. C'est l'exemple de facture la plus italique découvert jusqu'ici en Espagne.

- Mérida (mosaïque cosmologique), $c f$., en dernier lieu, notre art. $L a$ 
celle des Vents $\left({ }^{*}{ }^{15}\right)$. En revanche, la représentation du masque d'Océan dans un canevas géométrique, circulaire ou octogonal, où il est isolé de son contexte marin, semble y avoir connu une certaine vogue $\left({ }^{16}\right)$.

Avant d'aborder l'étude stylistique du masque d'Océan proprement dit, il nous faut dire un mot de l'iconographie des Vents choisie par le mosaïste de Faro. Jusqu'à présent, cinq mosaïques connues dans l'ensemble du monde romain associent Océan aux Vents $\left({ }^{17}\right)$. On les rencontre tout aussi fréquemment associés à des scènes dionysiaques $\left({ }^{18}\right)$, au cycle des Saisons $\left({ }^{19}\right)$, à un sujet cosmologique $\left({ }^{20}\right)$ qu'à la naissance de Vénus marine $\left({ }^{21}\right)$. Les Vents sont donc compatibles avec des sujets variés et leur représentation prend, dans la Péninsule Ibérique, au moins trois formes différentes: tête (vue de face ou de profil (fig. 7, 8 a) ou

mosaïque cosmologique de Mérida, étude technique et stylistique I, in «M.C.V.», XIX, 1983, p. 17-68.

Nous ne tenons pas compte dans cette liste de l'exemple d'Elche (Contra: A. Paulian, art. cité, reprenant la théorie de P. Palol, in El mosaico de tema oceanico de la villa de Dueñas, in «BSAA», 1963, p. 14 et 16): il ne présente pas, à notre avis, les traits caractéristiques du dieu Océan.

(15) Parmi les exemples cités supra n. 13, il faut peut-être mettre à part celui de la mosaïque cosmologique de Mérida, dont la composition plus complexe ne met pas exclusivement les Vents en relation avec Océan.

${ }^{(16)}$ C'est le cas dans les mosaïques de Balazote, La Quintanilla de la Cueza (les deux exemples), Cordoue (n. ${ }^{\circ}$ ) et peut-être à Milla del Rio, soit dans 4 ou 5 cas sur les 10 répertoriés en Espagne.

(17) Cf. supra n. 13.

(18) Dans la mosaïque de Bacchus de Baños de Valdearados (v. J. L. Argente Oliver, La villa tárdorromana de Baños de Valdearados, Madrid, 1979, pi. VII et fig. 18, la mosaïque de Cordoue (n. ${ }^{\circ}$ 2), la mosaïque de Mérida (in A. Blanco, Corpus de mosaicos de España, fase. I, Mérida, n. ${ }^{\circ} 43 \mathrm{~B}$, calle Masona).

(19) À Santa Vitoria do Ameixial, $c f$. M. Torres CARro, La escena de dises y las Sirenas del mosaico de Santa Vitoria, «BSAA», 1978, p. 89-102, pi. ï.

(20) Cf. M. H. Que t, La mosaïque cosmologique de Mérida, Paris, 1981, passim.

(21) Cf. A. M. Canto, El mosaico del nacimiento de Vénus en Itálica, «Habis», 7, 1976, p. 293-338. 
buste $\left({ }^{22}\right)$, en pied $\left({ }^{23}\right)$, avec ou sans attribut $\left({ }^{24}\right)$. Toutefois, la représentation la plus curieuse, et en même temps la plus tardive, est celle que l'on trouve dans la mosaïque du triomphe de Bacchus découverte à Baños de Valdearados: un chien de chasse courant après sa proie, et identifié comme un Vent par une inscription. On peut parler ici d'un abandon de la tradition au profit d'une interprétation libre du mosaïste du Vème s., qui ne dispose plus des modèles traditionnels de ces figures. À notre connaissance, il n'existe pas ailleurs de représentation semblable des Vents.

L'exemple de Faro, au contraire, illustre un type iconographique classique $\left({ }^{25}\right)$, bien connu jusqu'ici par les beaux exemples de la villa des Néréides à Tagiura $\left({ }^{26}\right)$ ou de la mosaïque de Sousse $\left({ }^{27}\right)$. Si on ne peut parler de fixité du type, il est tout-de-même frappant de voir le type le plus fréquent (la tête de profil) revenir du Ilème au IVème $\mathrm{s}$. L'exemple de Cordoue $\left({ }^{28}\right)$ où les Vents sont associés à un thème dionysiaque est isolé en Espagne mais on lui trouve un parallèle en Tunisie (mosaïque d'Oudna). La représentation des Vents, en pied, dans la mosaïque cosmologique de Mérida, situe ces divinités dans le contexte des grandes forces naturelles. On voit donc qu'il n'y a pas de correspondance

(22) Vent vu de face à Cordoue in Corpus de mosaicos de España, Córdoba, Jaén, Málaga, Madrid, 1981, n. ${ }^{\circ}$, et dans la mosaïque de la naissance de Vénus à Itálica. Vent vu de profil, dans les mosaïques de Faro, et de Santa Vitoria do Ameixial. Vent vu en buste, dans la mosaïque n. ${ }^{\circ} 43$ B du Corpus des mosaïques de Mérida, Madrid, 1978.

(23) Vent vu en pied: dans la mosaïque cosmologique de Mérida.

(24) L'attribut le plus courant est le souffle du Vent, matérialisé par un faisceau de lignes parallèles de couleur bleue ou verte, qui s'échappe de sa bouche, ou bien, comme dans la mosaïque de Milla del Rio, par une trompe marine que le Vent tient de son bras tendu (v. : T. MAÑanes, o. L, pi. XXVIII).

$\left({ }^{25}\right)$ Sur ce problème, v. l'ouvrage de K. Neuser, Anemoi, Studien zur Darstellung der Winde und Windgottheilen en der Antike, Rome, 1982, p. 175 sqq.

(26) Cf. A. Di Vita, La villa della gara delle Nereide a Tagiura, Supp, to «Libya antiqua», II, Rome, 1966, p. Vili, e, f.

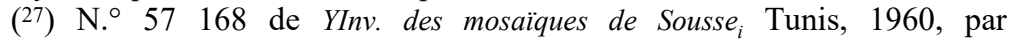
L. FOUCHER.

(28) V. réf. supra n. 18. 
entre le type choisi par le mosaïste et la fonction iconographique de ce Vent. Les types sont interchangeables, dans une certaine mesure, si l'on excepte le cas tout-à-fait à part des Vents dans la mosaïque de Baños de Valdearados; ce qui change, d'une mosaïque à l'autre, c'est la signification de l'association des Vents au reste des images du pavement $\left({ }^{29}\right)$.

Pour revenir aux Vents de la mosaique de Faro, on y observe une singulière simplification du type du Vent à tête vue de profil.

Si on les compare à ceux de la mosaïque d'Amphitrite, dans la villa de la course des Néréides à Tagiura, on constate qu'ils n'ont plus qu'une aile, que le buste au cou gonflé se réduit à la tête et au cou d'un volume normal, et que leur souffle n'est pas figuré. Par ailleurs, ils n'émergent pas des flots de la mer, comme le veut la légende, respectée par le mosaïste de Tagiura. On note également un manque d'expression dans les têtes des Vents de Faro, si on les compare à une représentation comparable, celle de la mosaïque des philosophes découverte Piazza della Vittoria à Paierme $\left.{ }^{(30}\right)$.

Il y a donc un contraste entre leur association légitime avec le dieu de la mer, d'où naquirent les Vents, et la représentation assez sèche de ces dieux, dans la mosaïque de Faro.

\section{Le masque (TOcéan (fig. 6)}

La technique utilisée par le mosaïste est celle d'un pseudo-emblema, comme nous l'avons vu supra. L'usure des tesselles de verre, que nous avons signalée s'explique peut-être, dans ce cas particulier - la mosaïque ornait peut-être une salle de thermes publics, ou de tout autre édifice lié à l'activité maritime (bourse de commerce, halle au poisson, schola de corporation ?) — par l'usage intensif du sol mosaïqué.

$\left.{ }^{29}\right)$ Cette particularité laisse supposer que les Vents constituaient des modèles indépendants, interchangeables avec d'autres motifs classiques dans les angles, comme les Saisons.

$\left.{ }^{30}\right) C f$. en dernier lieu, l'ouvrage de D. von Boeserager, Antike Mosaiken in Sizilien, Rome, 1983, p. 176-183, et pi. LXI, LXII. 
Cette destination publique de l'édifice explique sans doute le choix des commanditaires, c.à.d. la version la plus géographique $\mathrm{du}$ sujet. Océan, père de toutes les eaux ${ }^{(31}$ ) qui fait naître les Vents, dont le souffle favorise la navigation, le commerce. Cosmologie et géographie humaine inspirent cette mosaïque $\left({ }^{32}\right)$ et non la mythologie ou la scénographie $\left.{ }^{33}\right)$. On n'a pas non plus ici une tête simplement décorative, comme le masque d'Océan peut le devenir dans des contextes moins marqués $\left({ }^{34}\right)$. Le dieu n'a pas non plus à Faro le regard terrible (sidereo visu) qu'on lui prête dans certaines de ses représentations tardives ${ }^{(35)}$.

Le modèle suivi par le mosaïste de Faro se caractérise par un expressionnisme mesuré: c'est un homme grave, aux traits réguliers, sans recherche excessive du pathétique. Le flot qui coule de ses lèvres se réduit à une ou deux lignes de tesselles. De même, ses attributs sont figurés sans exagération dans les proportions; les couleurs choisies pour rendre son teint sont naturalistes, et non arbitrairement choisies pour provoquer la terreur, comme dans l'exemple célèbre d'Ain-Temouchent.

Le style de ce masque invite donc à le situer chronologiquement vers la fin du Ilème s. ou le début du Illème s.

(31) Dans son article Notes sur Viconographie d'Océan, "MEFRA», 84, 1972, p. 631-673, P. Voûte a bien mis en évidence ce trait fondamental du dieu.

(32) Comme dans la mosaïque du catalogue de navires d'Althiburos (Maison des Muses) in K. M. D. Dunbabin, Roman mosaics from North Africa, Londres, 1978, p. 127 et 153, datée delà deuxième moitié du Illèmes. Pour toutes les mosaïques africaines illustrant ce sujet, nous renvoyons également au livre de K. M. D. Dunbabin, dont l'excellent catalogue facilite les recherches bibliographiques. Pour les mosaïques avec représentation d'Océan, v. p. 149-154.

(33) Comme dans la mosaïque de Dueñas, et la plupart des pavements africains où Océan apparaît dans le contexte d'un cortège marin.

(34) Par exemple dans les thermes d'Océan à Sabratha (in S. AuRIсем ма, L'Italia in Africa, Tripolitania, I monumenti d'arte decorativa, I mosaici, Rome, 1960, pi. 3.

(35) Comme dans la mosaïque d'Ain-Temouchent (in K. M. D. Dunвав гn, o. /., pi. 143, p. 151, la mosaïque d'El Haouna et de Dermech (ibid., p. 107) et la mosaïque de Themetra (ibid., pi. 144). Selon K. M. D. Dunbabin, c'est un trait des mosaïques d'Océan au IVème s. 
Depuis la parution des études de L. Foucher ( $\left.{ }^{36}\right)$, P. Voûte $\left({ }^{31}\right)$, A. Paulian (14) et K. M. D. Dunbabin ( ${ }^{32}$ ), l'éventail des mosaïques d'Océan connues s'est légèrement élargi $\left({ }^{37}\right)$. Le principe de classification retenu par L. Foucher en 1963 — c.à.d. l'emplacement du masque dans la mosaïque - ne paraît plus pertinent, comme il le reconnaît lui-même, implicitement, dans son article consacré au même sujet, en 1975 .

(36) Dans son ouvrage La maison de la procession dionysiaque à El Jem, Paris, 1963, p. 143-145, et Id. Sur Viconographie du dieu Océan «Caesarodunum», n. ${ }^{\circ} 10,1975$, p. $48-52$, qui n'apporte pas de modifications substantielles à l'étude précédente.

(37) Pour la Péninsule Ibérique, v. n. 14. En Italie, deux exemples nouveaux:

mosaïque d'Ancône, signalée dans les F.A., XVI, 1961 (1964), par G. Annibaldi, sous le n. ${ }^{\circ}$ 4151. Il s'agit d'un pseudo-emblema, daté du Illème s. Sur le même pavement, v. également: H. BLAnck, "AA.», LXXXVI, 1971, p. 312, fig. 52; et une bonne photographie en couleurs dans $V E A A$ s.v. Ancona, Supp.t 1970, en face de la page 56.

mosaïque des Horrea Agrippiana sur le Forum, publiée par F. Astolfi, F. Guidobaldi, A. Pronti dans "Arch. Class.», XXX, 1978, p. 31-100 et pi. XXXVIII (mosaïque en noir et blanc, de l'époque des Antonins ou du début du Illème s., d'après les A.). Enfin, il faudrait compléter la bibliographie sur la mosaïque d'Océan connue depuis longtemps à Piazza Armerina, mais sur laquelle sont revenus récemment A. Ricci et M. DE Vos dans Filosofiana, la villa de Piazza Armerina, Paierme, 1982, p. 258 et feuillet XXXVIII.

En Gaule: mosaïque de Maubourguet, in C. Balmelle, S. Doussau, La mosaïque à l'Océan trouvée à Maubourguet, "Gallia», 40, 1982, p. 149-170. Cette mosaïque est postérieure au IVème s.

Pour les autres pavements:

d'Aquitaine, $v$. C. Balmelle, Recueil, IV, 1, n. ${ }^{\circ} 154$ (Jurançon),

de Lugdunaise, v. J. P. Darmon, H. Lavagne, Recueil, II, 3, n. ${ }^{\circ} 468$.

de Narbonnaise, v. J. Lancha, Recueil, III, 2, n. ${ }^{\circ} 277,308$ et 393, de la deuxième moitié du lind s..

En Grande-Bretagne, mosaïque de Dorchester, in J. M. C. ToynB E E, Art in Roman Rritain, Londres, 1962, n. ${ }^{\circ} 178$.

mosaïque de Woodchester, ibid. p. 267-273 et pi. LXII, b.

mosaïque de Rudston (York) in D. S. NE aL, Roman Mosaics in Rritain, Gloucester, 1981, n. ${ }^{\circ}$ 67, p. 94 (milieu du IVème s.).

En Allemagne, une nouvelle étude de $\mathrm{H}$. Bullinger sur la mosaïque de Bad-Kreuznach : Die Verwendung von farbigen Glas in zwei Mosaiken des 3 Jaht hundert n. Ch. aus Rad-Kreuznach, in "Actes du Vllème Congrès de VAssociation internationale de Vhistoire du verre», Berlin, 1977, p. 41-49. 
Les recherches plus récentes ont justement mis l'accent sur la signification réelle de ce masque dans les mosaïques.

Dans la mesure où ces pavements sont désormais datés avec un peu plus de certitude qu'ils ne pouvaient l'être dans les années 60, on est amené à distinguer en fait deux grands groupes chronologiques, plus larges que ne l'envisageait L. Foucher. Jusqu'au Ilème s., le modèle courant représenté avec plus ou moins de fidélité et d'expressivité est de caractère pictural $\left({ }^{38}\right)$ : couleurs naturalistes et attributs fixes. C'est à ce type, nous l'avons vu, qu'appartient l'Océan de la mosaïque de Faro. Les mosaïques qui nous paraissent les plus représentatives de cette série sont le masque d'Ouzoüer-sur-Trézée ${ }^{39}$ ) et celui découvert à Ancône, bien que ces exemples ne présentent pas tous les attributs canoniques énumérés par P. Voûte dans son excellente étude du sujet. Il nous semble que la fidélité à un modèle pictural est l'un des éléments à prendre en compte également $\left({ }^{40}\right)$. D'autre part, une seconde distinction doit être faite entre le masque du dieu et les représentations d'Océan en dieu fleuve, comme c'est le cas dans la mosaïque cosmologique de Mérida et dans deux mosaïques d'Antioche. Il s'agit alors d'un autre modèle, d'origine orientale, et dont la mosaïque de Mérida fournit la seule illustration dans les provinces occidentales.

Nous avons énoncé ailleurs ( $\left.{ }^{41}\right)$ l'hypothèse d'une origine africaine du type iconographique du masque d'Océan, dans la mesure

(38) Un bel exemple en peinture dans le rnithraeum de Santa-Maria Capua Vetere, $c f$. M. Vermaseren, The Mithraeum at Santa-Maria Capua Vetere, «EPRO», 16, 1971, p. 8 et pi. VII. M. Vermaseren remarque à juste titre que le dieu Océan y est représenté sous les traits d'un homme jeune, plein de vitalité, et non d'un vieillard, comme c'est le cas dans les mosaïques tardives africaines.

(39) Nous suivons sur ce point l'opinion de H. Stern et J. P. Darmon, in Recueil, II, 3, n. ${ }^{\circ}$ 468. Contra: G. Сн. PICARD, «CMGR», I, qui voit dans cette tête une tête de Saturne.

(40) A ce sujet, nous écartons comme masque d'Océan les figurations de dieux fleuves que l'on trouve sur les monnaies citées par A. Paulian, art. cité, p. 123. Dans le même ordre d'idées, la datation avancée par Ch. Picard pour le masque de bronze de Lixus - le règne de Juba II — ne nous semble reposer sur aucune donnée archéologique.

(41) In Recueil, III, 2, n. ${ }^{\circ} 277$, Observations. 
où les illustrations du sujet les plus nombreuses connues à ce jour sont en Afrique du Nord (une vingtaine). La mosaïque d'Ancône attribuée au Illème s. constitue le seul exemple du sujet traité à la fois en polychromie et en bichromie, selon des critères picturaux: il ne s'agit pas d'un démarquage des silhouettes en noir et blanc. En attendant la publication du corpus de la région des Marches, qui permettra de situer ce pavement dans son contexte régional - ou de l'attribuer à un artiste itinérant - , il nous faut constater que, aussi bien en noir et blanc qu'en polychromie, aucune mosaïque n'illustre ce sujet avant l'époque d'Hadrien. Il en va de même pour les illustrations en peinture ou en sculpture.

Un classement chronologique des mosaïques illustrant ce sujet dans la Péninsule Ibérique fait apparaître une mosaïque datée du IVème s. pour neuf datées du Ilème et du Illème s. $\left({ }^{42}\right)$.

Cette apparente continuité ne va pas sans quelques variantes:

- le masque végétal, qui n'a plus rien du masque original, dans la mosaïque de la pièce $n .^{\circ} 24$ de la villa de Quintanilla de la Cueza, en Vieille Castille, loin de la mer.

- le masque stylisé à l'excès, avec perte des éléments caractéristiques, comme la direction et l'expression du regard. $\mathrm{Au}$ contraire, on remarque un ajout curieux dans la mosaïque $n .^{\circ} 2$ de Cordoue (14) qui ne fait pas partie du masque traditionnel: bonnet du genre pilos? ou sorte de casque par contamination avec les masques végétaux?

En Espagne, on ne peut faire que des suppositions sur l'introduction du modèle africain du masque traditionnel d'Océan. La proximité de l'Afrique fournirait un argument séduisant; et l'exemple de Faro, si proche des modèles africains du Ilème et Illème s. par son style pictural, fournirait un argument en faveur de cette thèse.

${ }^{42}$ Dans le premier groupe (Ilème, Illème s.) se trouvent les mosaïques de Milla del Rio, Cordoue, Lugo, Mérida, Quintanilla de la Cueza, Balazote, dans le second se trouve la mosaïque de Dueñas. 
Enfin, dans un autre ordre d'idées, on peut se demander si l'association du masque d'Océan avec le décor végétal des hexagones est arbitraire ou significatif. Peut-être faut-il y voir une allusion à la fertilité provoquée par les eaux d'Océan? Nous n'avançons cette hypothèse qu'avec une extrême prudence, car les compagnons habituels du dieu, quand ils sont figurés, sont toujours les poissons et autres êtres marins.

\section{L'inscription}

Caractères intrinsèques (figs. 11, 12):

Inscrite dans un cadre dont la forme imite celle d'une tabula ansata, de 2,45 X 0,42 m, en lettres noires sur fond blanc, de $0,10 \mathrm{~m}$ de haut, elle comporte trois lignes, avec une lacune centrale de $450 \mathrm{~mm}$ de long à la 1ère ligne, $480 \mathrm{~mm}$ à la 2ème ligne, et $620 \mathrm{~mm}$ à la troisième. Comme font noté les restaurateurs au moment de la dépose, les lettres avaient été préalablement dessinées à la peinture rouge sur la couche supérieure du nucleus.

On note une certaine irrégularité dans l'espacement des lettres selon les lignes. Aux lignes 1 et 2 , il est assez régulier, et la coupure est faite normalement à la syllabe, en fin de première ligne. De manière générale, les lettres sont plus hautes que larges. Les noms des personnages sont mis sur le même plan, du point de vue de l'espace qu'ils occupent. Au contraire, à la troisième ligne, on remarque une certaine négligence dans l'écriture et la composition: au resserrement des lettres en début de ligne succède le verbe donarunt, qui se détache nettement du reste par l'espacement et les dimensions des lettres.

Bien que dessinées avant d'être exécutées, les lettres n'ont pas le bel aspect des capitales carrées des inscriptions monumentales sur pierre. Les jambages ne sont pas tous droits.

L'interponction est syllabique, mais placée de manière fantaisiste, presqu'au pied de la lettre, ou à mi-hauteur, et sans régularité. Les tesselles blanches du fond ont été visiblement posées après l'exécution des lettres, ou plutôt au fur et à mesure que chaque mot ou chaque groupe de mots était écrit en tesselles 
noires: le même lit de pose était utilisé pour les lettres et le fond blanc.

Enfin, pour une lettre comme le $\mathrm{C}$, on observe une graphie hésitant entre le $\mathrm{C}$ et le $\mathrm{G}$ (C. Calpurnius, C. Vibius) tandis que le $\mathrm{G}$ (dans Geminus) est écrit comme un C.

\section{Commentaire}

Des quatre personnages désignés par leur praenomen, nomen, cognomen, trois portent des noms trop répandus pour pouvoir donner lieu à une recherche d'identification (C. Calpurnius, C. Vibius Quintilianus et C. Attius). Tout au plus peut-on remarquer qu'aucun d'eux ne porte un surnom terminé en -ius; une datation au IVème s. est donc exclue.

La restitution proposée par J. d'Encarnaçâo, à la ligne 2, - C. Att[ius] - est pratiquement sûre. Le nomen du quatrième donateur - Verrius - est plus rare et peut mettre sur la piste d'une famille dont le rôle social dans la province de Lusitanie, ou en Bétique toute proche, est plus facile à cerner $\left({ }^{43}\right)$. Ce gentilice est connu en effet par deux inscriptions peintes sur des amphores découvertes à Rome, et provenant de Bétique. Il s'agit des n. 250 (dans M. Beltrán Lloris, Las ánforas romanas en España, Saragosse, 1970) L. Verri Apri, et 251 (ibid.) Verriorum. D'après M. Beltrán, les Verrii furent exportateurs pendant une longue période, dans la seconde moitié du Und s.

A la ligne 3 , nous avons proposé ailleurs $\left({ }^{44}\right)$ de restituer:

\section{tessella s[ternere curar]unt et donarunt.}

Toutefois, ayant procédé par la suite à des mesures plus précises, et compte-tenu du resserrement des lettres à cette ligne, jusqu'au

$1^{43}$ ) Nous adressons à Sylvie Dardaine nos remerciements les plus vifs pour ses critiques et suggestions dans le domaine épigraphique.

(44) Cf. notre article, Les mosaïstes dans la vie économique de la Péninsule Ibérique, du ler au IVème s.: état de la question et quelques hypothèses, «M.C.V.», XX, 1984, p. 45-61. Cf. également la notice n. ${ }^{\circ} 490$ de l'Année Épigr., 1981, où <st proposée la lecture «solum tessellatum», et la date du Illème ou du IVème $s$. 
mot donarunt, nous proposons la lecture suivante, qui conserve la construction - tessella sternere-:

solum tessella s[ternendum curar] unt et donarunt.

La fonction des quatre personnages n'est pas précisée dans l'inscription, mais son caractère officiel — d'une nature qui reste à déterminer - ne fait aucun doute. S'agit-il des quattuor viri d'Ossonoba? ou de responsables d'un collège professionnel ou religieux? Le fait qu'il y ait quatre noms exclut en tout cas qu'il s'agisse d'une maison privée. Nous avons mentionné dans l'article cité supra notre préférence pour la première des hypothèses faites par J. d'Encarnaçâo, c.à.d. pour celle qui voit dans ces personnages les quattuor-viri d'Ossonoba, à une date qu'il est malheureusement impossible de fixer avec précision. Certes, les données disponibles aujourd'hui sur l'administration locale en Lusitanie sont assez minces. Sur les 38 inscriptions découvertes dans la ville ${ }^{45}$ ) la plupart sont funéraires, et deux inscriptions seulement nous font connaître un flamine municipal (CIL II n. ${ }^{\circ}$ 5141): C. Iulius Felicior, et un seoir augustal (CIL II n. ${ }^{\circ} 5133$ ): M. Cornelius Eridanus. Par ailleurs, R. Étienne a réuni quelques données éparses sur l'histoire d'Ossonoba $\left({ }^{46}\right)$. Elles nous permettent seulement d'entrevoir le déroulement de la vie de ce municipe à l'époque impériale.

Si l'on essaie enfin d'éclairer cette dédicace par des rapprochements avec d'autres dédicaces similaires sur des pavements, on est frappé du petit nombre des exemples conservés, tant dans l'espace que dans le temps. Du Und s. avant J. C. au Illème s. ap. J.C., dans l'ensemble des provinces occidentales de l'Empire, on ne trouve que quatre exemples, deux inscriptions certainement

$\left.{ }^{43}\right)$ F. CIL II, n. ${ }^{\circledR} 1$ à 12 et $C I L$ II Suppl. n. ${ }^{\circ} 5133$ à 5159.

(46) Dans son ouvrage Le culte impérial dans la Péninsule Ibérique, Paris, 1958, p. 223 (le municipe à'Ossonoba), 200, 254, 311, p. 502 (le buste de Gallien découvert à Ossonoba), p. 515 (un évêque d'Ossonoba au concile d'Elvira). 
signées par des magistrats $\left({ }^{47}\right)$ et deux par de simples particuliers $\left({ }^{48}\right)$.

Trois sur quatre de ces inscriptions, aussi éloignées soient-elles dans le temps et par leur contexte, adoptent la présentation formelle de la tabula ansata, claire référence à Pépigraphie officielle sur pierre. N'est-ce-qu'une coïncidence? ou bien l'indication du caractère officiel de l'inscription? il peut ne s'agir en fait que d'une imitation (dans le cas de Marcunus et de Calpurnius) un peu vaniteuse des grandes inscriptions publiques de la part d'un modeste propriétaire ou évergète. Mais ce ne sont là que diverses hypothèses, étant donné que l'on ne connaît pas avec certitude

(47) À Rome, l'inscription du temple d'Apollon Sosien, mosaïque noire et blanche commémorant l'oeuvre des édiles ( $c f$. DEgrassi, Inscriptiones latinae liberae Rei Publicae. Imagines, Rome, 1965, n. ${ }^{\circ}$ 26). À Alba Fucens, une inscription en lettres blanches sur fond noir, où on lit Vibia C. F. Galla balnea de sua pecunia ref. curavit, cf. «Ant. Class.», XXX, 1961, p. 67, 129. Il s'agit de la fille de l'empereur C. Vibius Trebonianus qui offre de ses deniers la réfection des thermes de la ville. En Espagne, une inscription sur un sol en terrazzo-signinum à Mazarrén (Murcie), cf. notre article cité n. 44 , p. 49, et en dernier lieu, la communication de B. Ramallo au colloque d'épigraphie de Saragosse (1983), où il opte pour l'identification de ces magistrats avec les magistri argentarii, fonctionnaires romains chargés de l'exploitation des mines d'argent situées à La Herrería.

(48) En Suisse, un exemple à Avenches ( $c f$. Y. von Gonzenbach, Die römischen Mosaiken der Schweiz, Bâle, 1960, p. 72 et pl. 26); un exemple dans le sanctuaire rural découvert près du Fort Trigh Tarhùna, en Tiipolitaine ( $c f$. S. Au rigem ма, Vitalia in Africa, I monumenti d'arte decorativa, / mosaici, Rome, 1960, p. 37 et pl. 56, où on lit:

\section{[CJALPVRNIVS \\ CANDİ DYS'VS}

.D. . D .

Enfin, il faut citer, en Germanie, la mosaïque de Victorinus à Trêves (in K. Parlasca, Die Römischen Mosaiken in Deutschland, Berlin, 1959, p. 44 et pl. 48.5) où on lit:

\section{PIANIVS VICTO/RINVS TRIBVNVS P/RET[ORIA]NORVM D[E SVO R]ESTITVIT.}

La mosaïque date du milieu du Illème s. 
la destination des deux édifices en question: un sanctuaire rural dans le cas de l'inscription de Tripolitaine, dans le portique d'un édifice public à Avenches ( ${ }^{49}$ ).

Dans l'état actuel de nos connaissances, on ne peut donc que souligner l'intérêt exceptionnel de l'inscription sur mosaïque de Faro: les quatre personnages du municipe d'Ossonoba qu'elle nous fait connaitre sont probablement des quattuor-viri, ou bien d'autres personnages exerçant des responsabilités professionnelles ou religieuses. Dans tous les cas, en demandant au mosaïste d'immortaliser leur don sur cette inscription, ils font preuve de civisme, ou manifestent leur évergétisme.

On voit donc que, par le sujet et l'inscription qui l'accompagne, la mosaïque d'OSsonoba nous confirme le degré de romanisation $\mathrm{du}$ municipe, et son ouverture aux courants artistiques venus d'Afrique, dans le domaine de la mosaïque.

(49) Dans une mosaïque . découverte en Angleterre au siècle dernier, une inscription sur mosaïque du même type accompagne un pavement dont deux bordures successives seulement sont dessinées. L'ensemble n'est connu que par un dessin. Il s'agit d'une mosaïque ornant la cella d'un temple - d'après l'inventeur-, situé à Lydney Park (in W. Hiley BAthurst, Roman Antiquities at Lydney Park, Gloucester, Londres, 1879, p. 25, p. VIII). On lit:

D(co) M(aximo) IT(erum) FLAVIVS SENILIS PR(aeses) REL(igionis) EX STIPIBVS POSSVIT (opitu)LANTE VICTORINO INTER(prete) (La)TINO.

Le point commun entre cette inscription et celle de Faro est évident: dans les deux cas, un personnage ayant une fonction officielle a pris en charge la construction d'un édifice et son décor. Toutefois, dans la mosaïque de Lydney Park, on précise l'origine du financement: ex stipibus. 


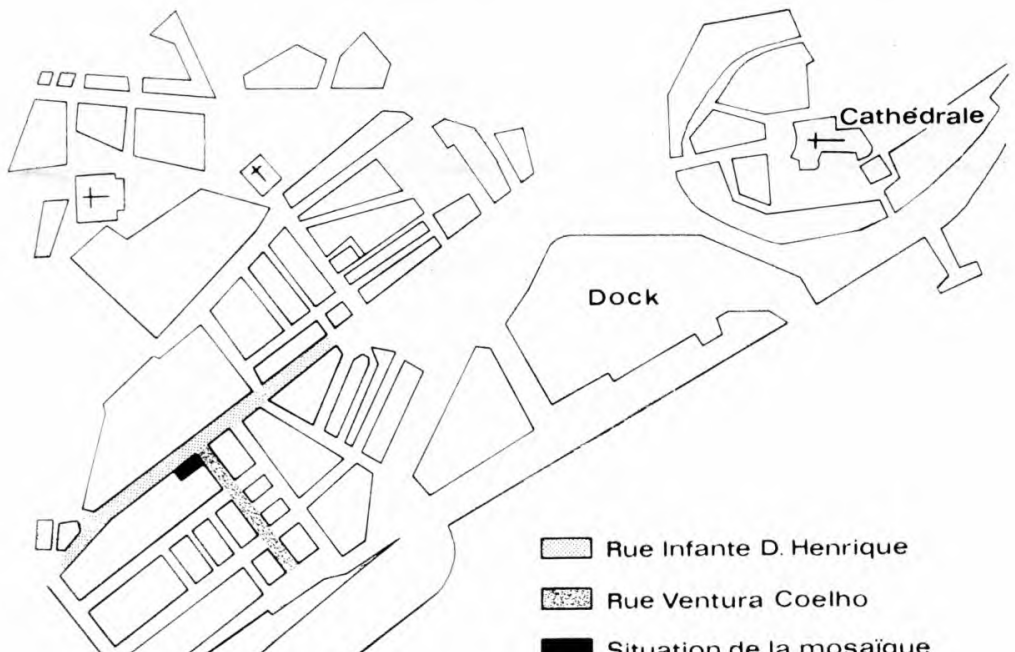

Situation de la mosaïque

FIG. 1 - Plan de situation. 


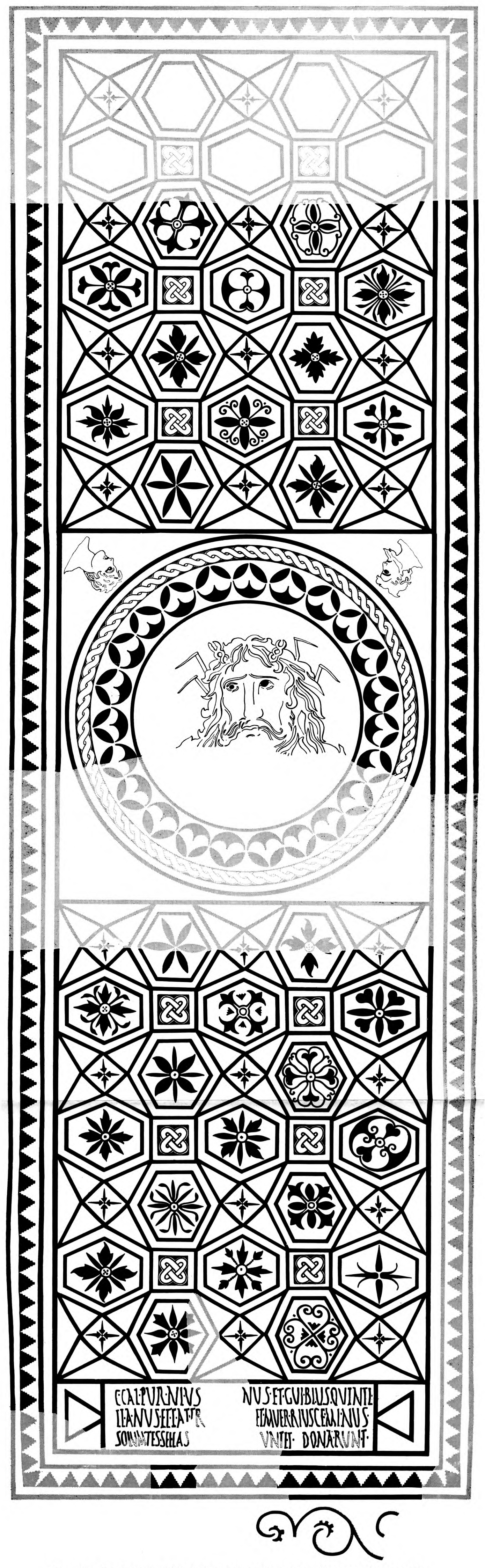


(Página deixada propositadamente em branco) 


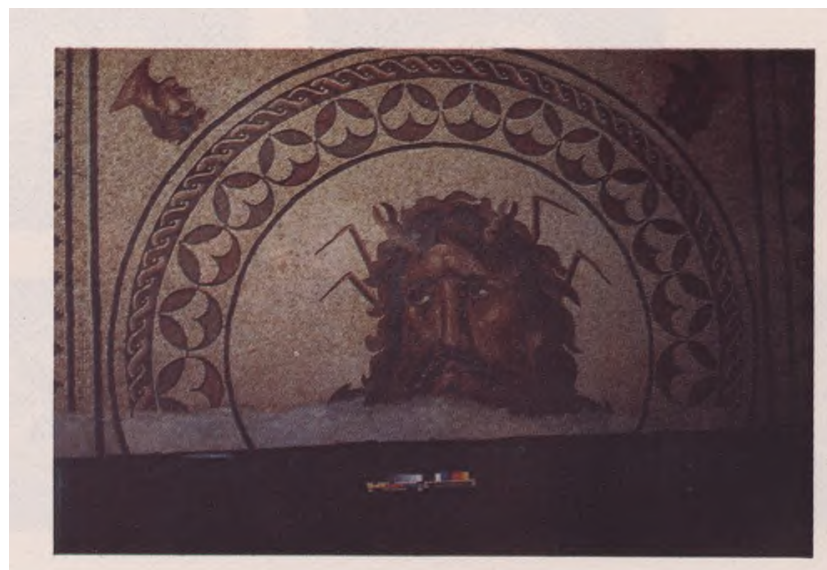

Fig. 3. - Vue du tableau central de la mosaïque d'Océan

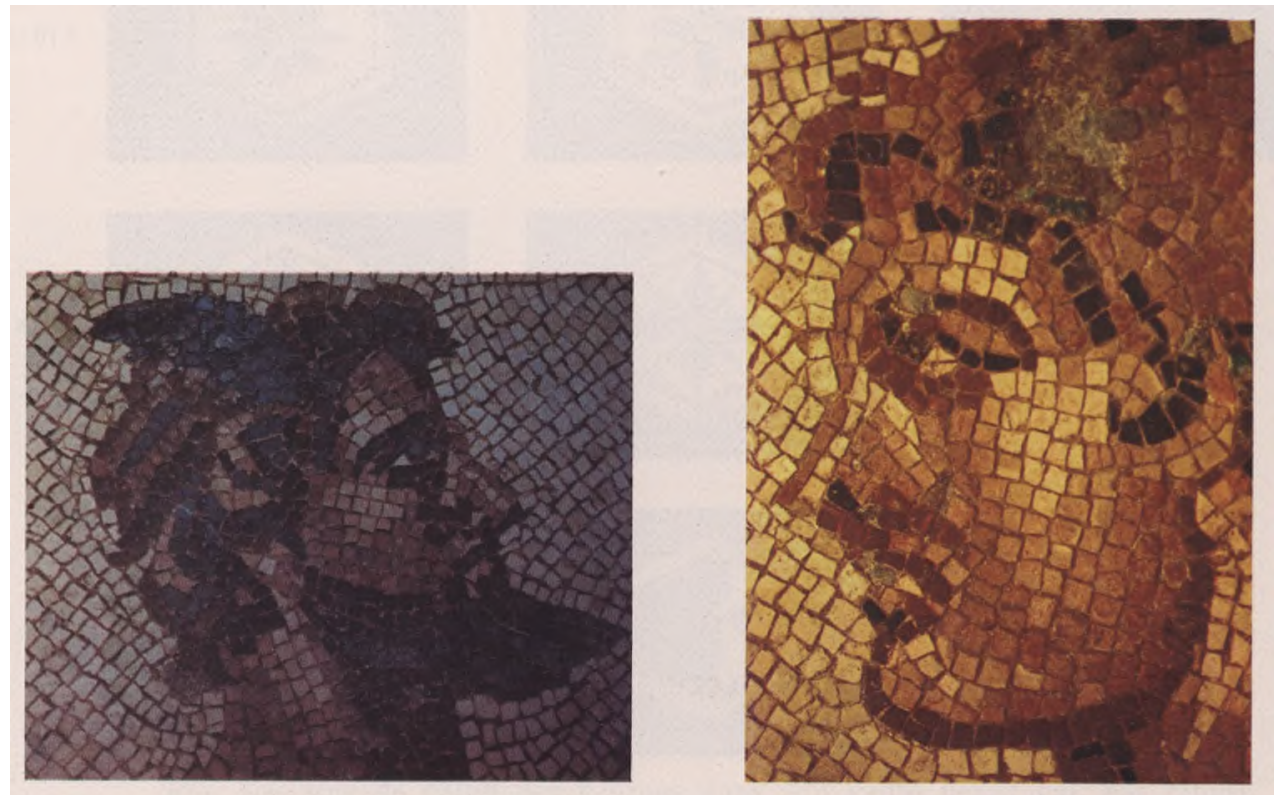

Fig. 5.-Détail des Vents de la mosaïque de Faro: 5a: Eurus (?); b: Boreas 
(Página deixada propositadamente em branco) 
III a; b
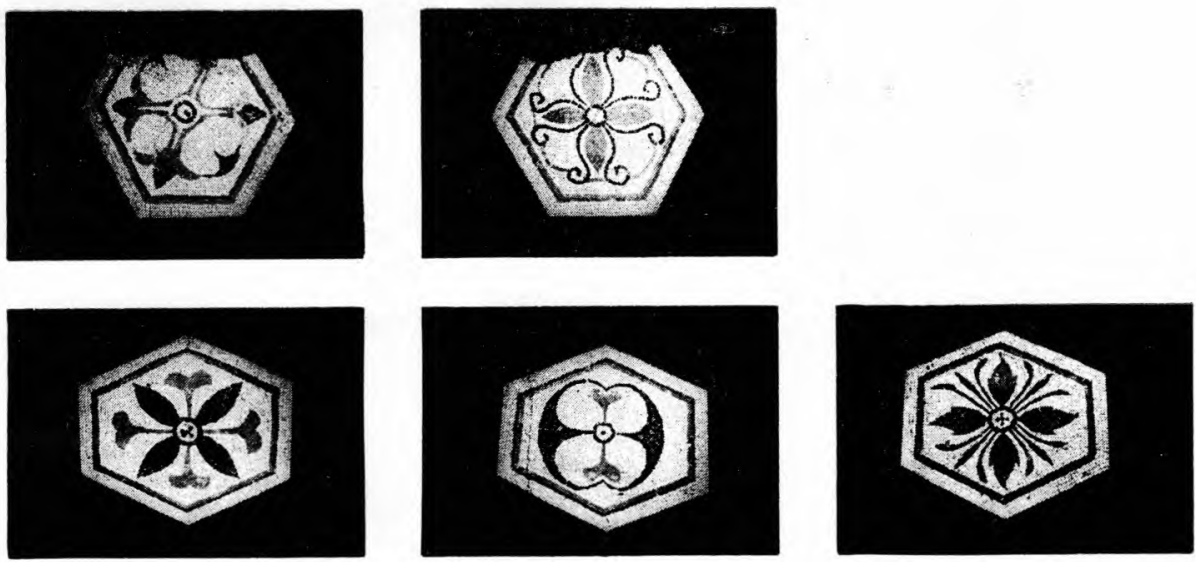

IV $a ; b ; c$
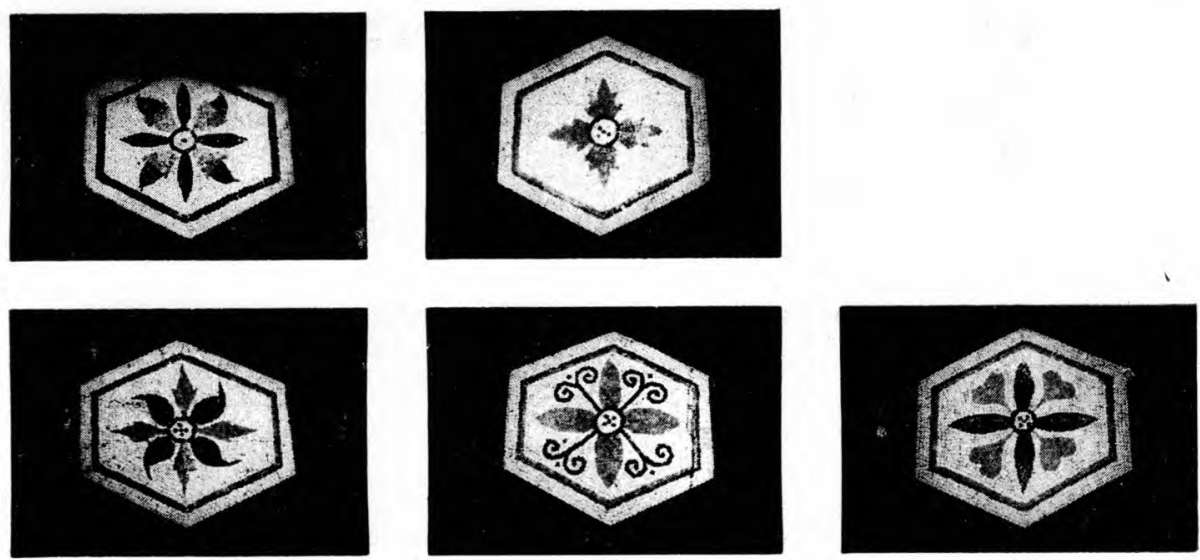

VII $\mathbf{a} ; \mathbf{b}$
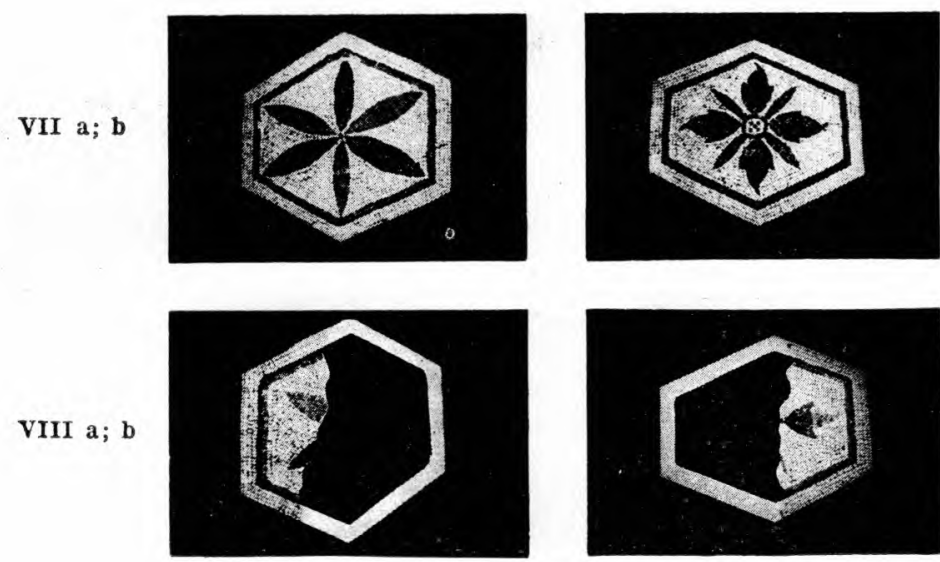

Fig. 4-Vue de détail des fleurons dans leur cadre hexagonal, par rangée de gauche à droite 
IX a; b ; c
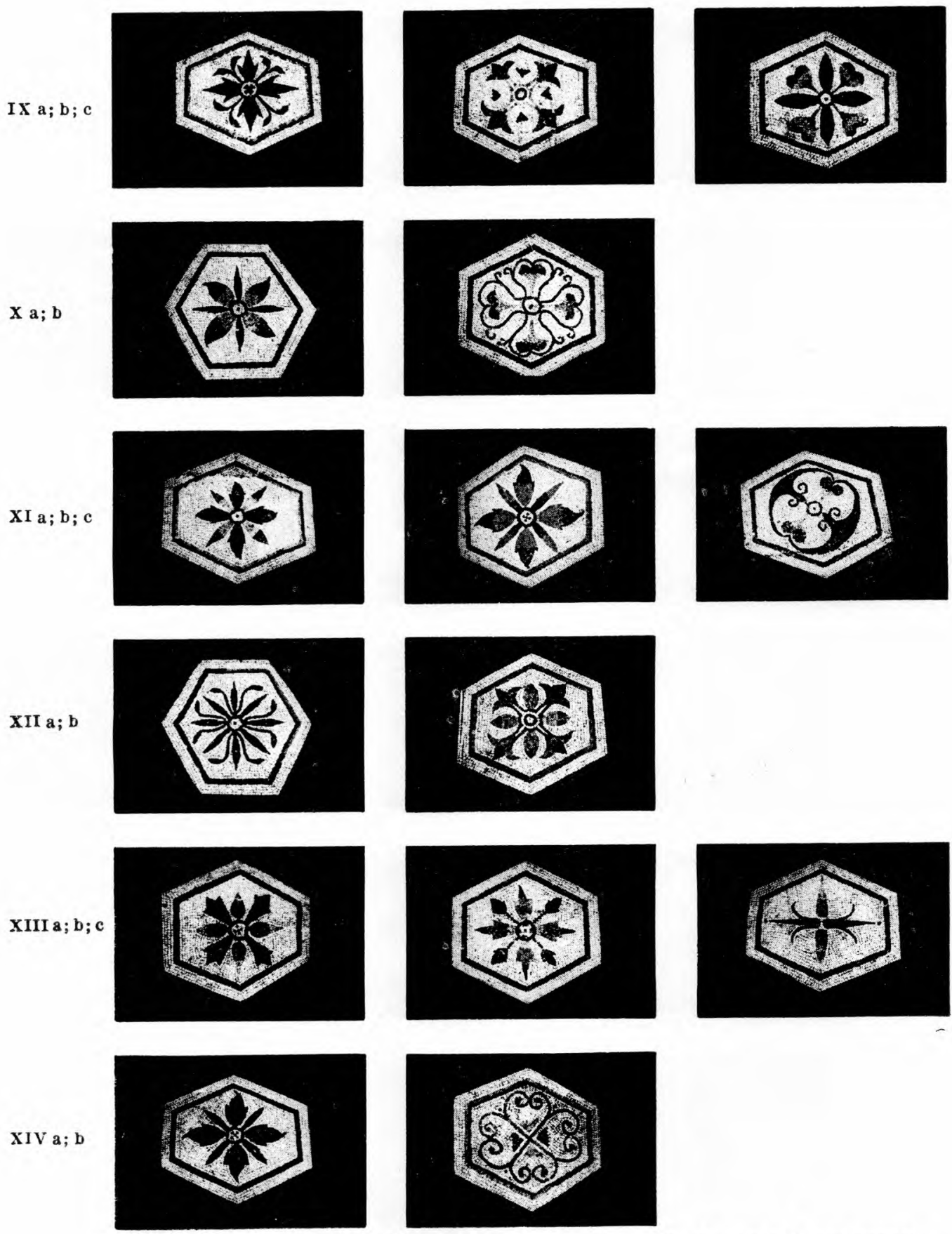

Fig. 4a-Vue de détail des fleurons dans leur cadre hexagonal, par rangée de gauche à droite 


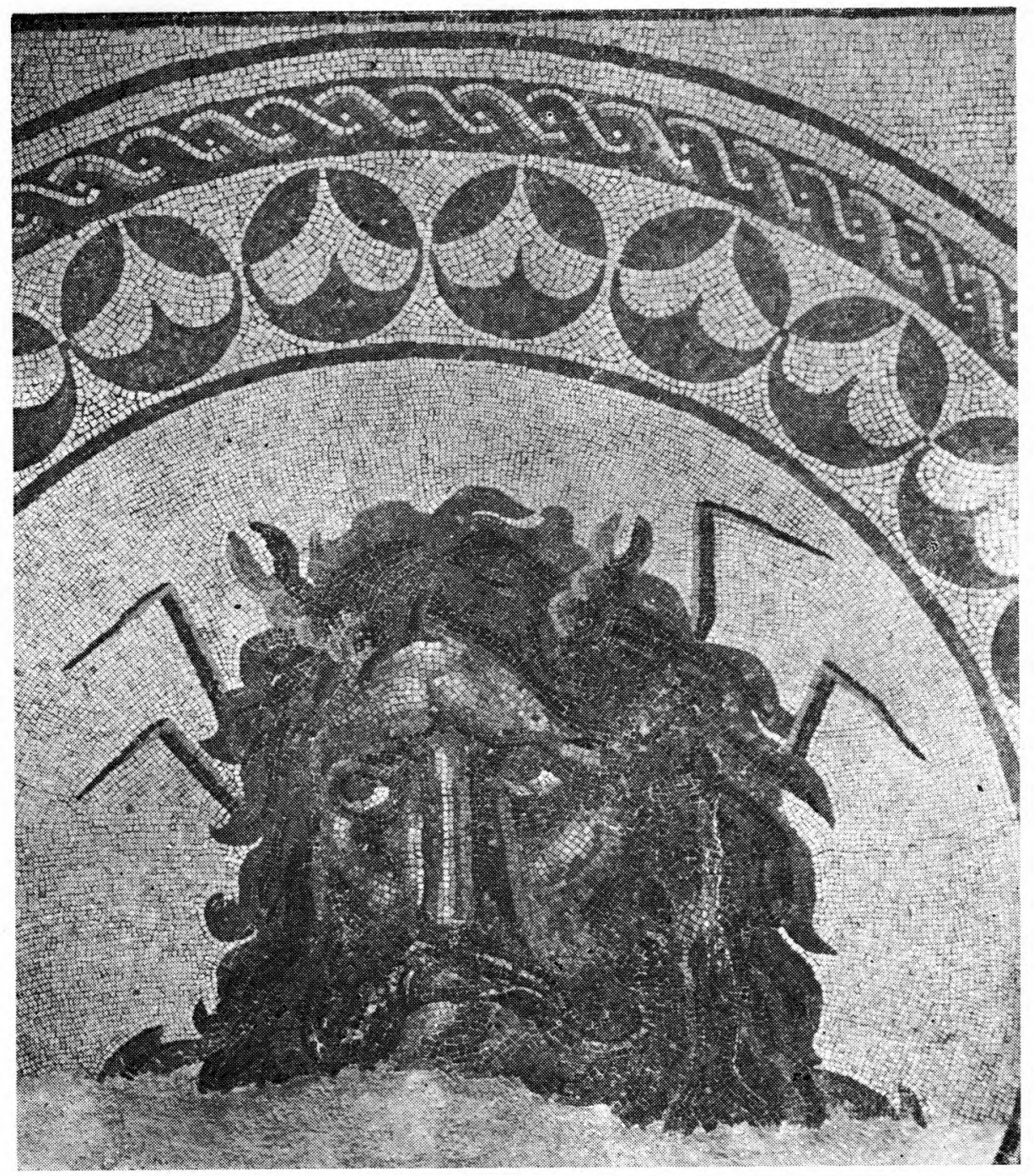

FIg. 6-Vue de détail du masque d'Océan 


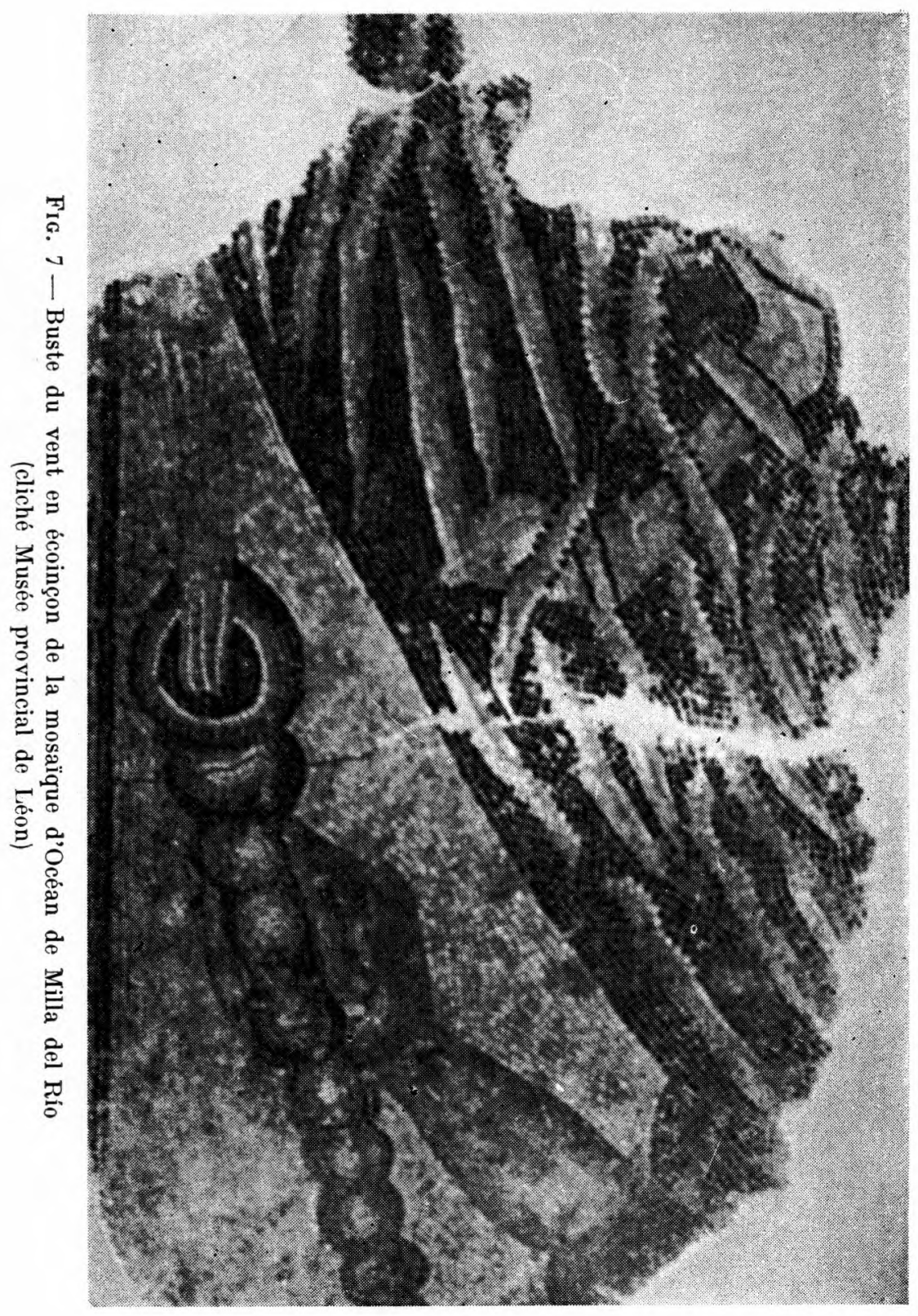




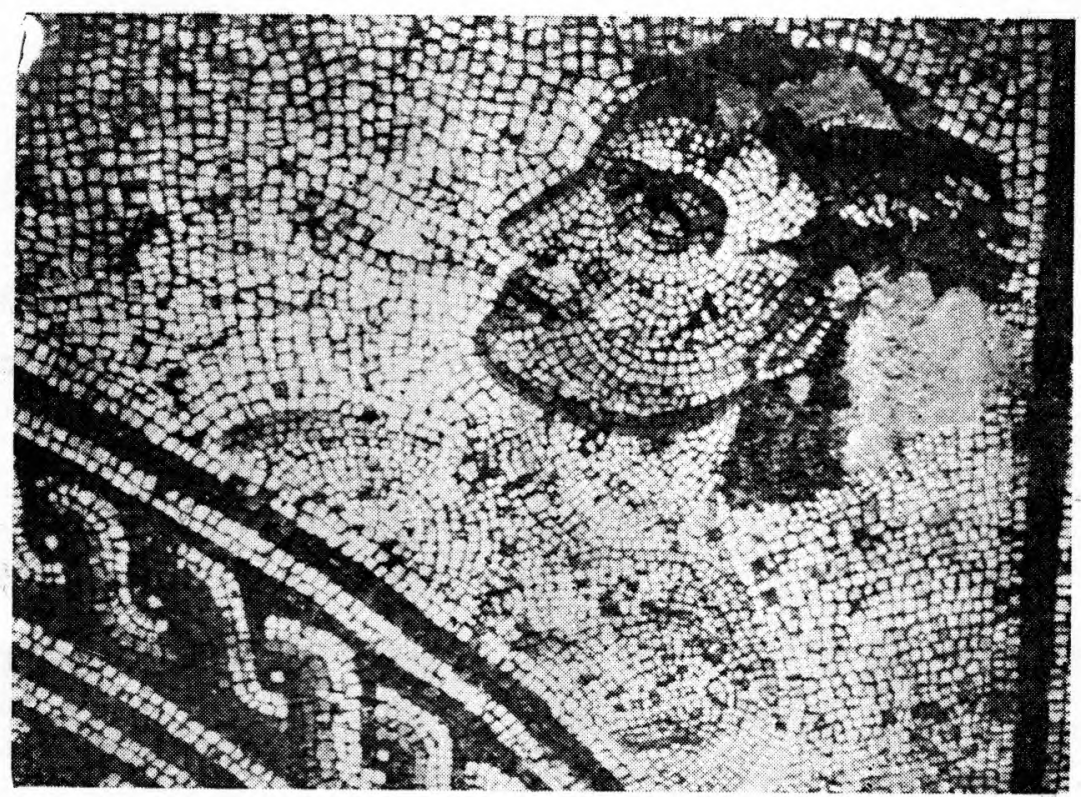

Fıg. 8a -.-. Vent de la mosaïque de Balazote, au Musée d'Albacete.

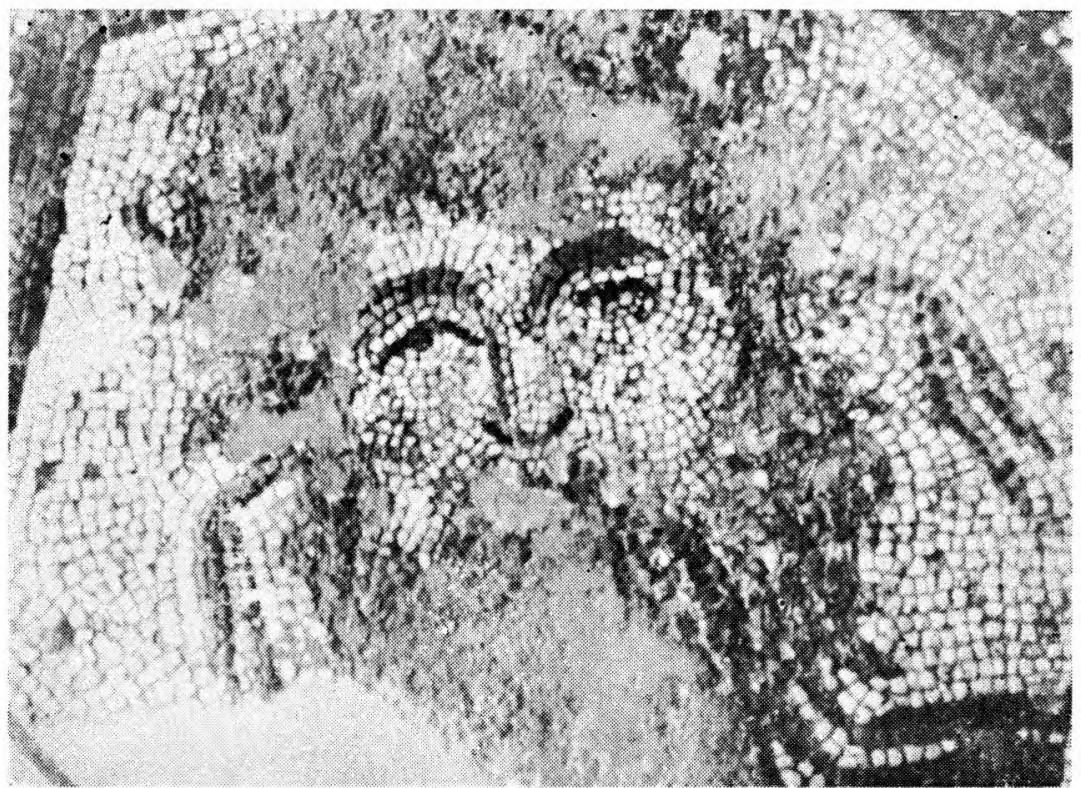

Fıg. 8b - Océan de la mosaïque de Balazote, au Musée d'Albacete. (Cl. de l'A. avec l'aimable autorisation de S. Samuel de los Santos, alors Directeur du Musée d'Albacete 


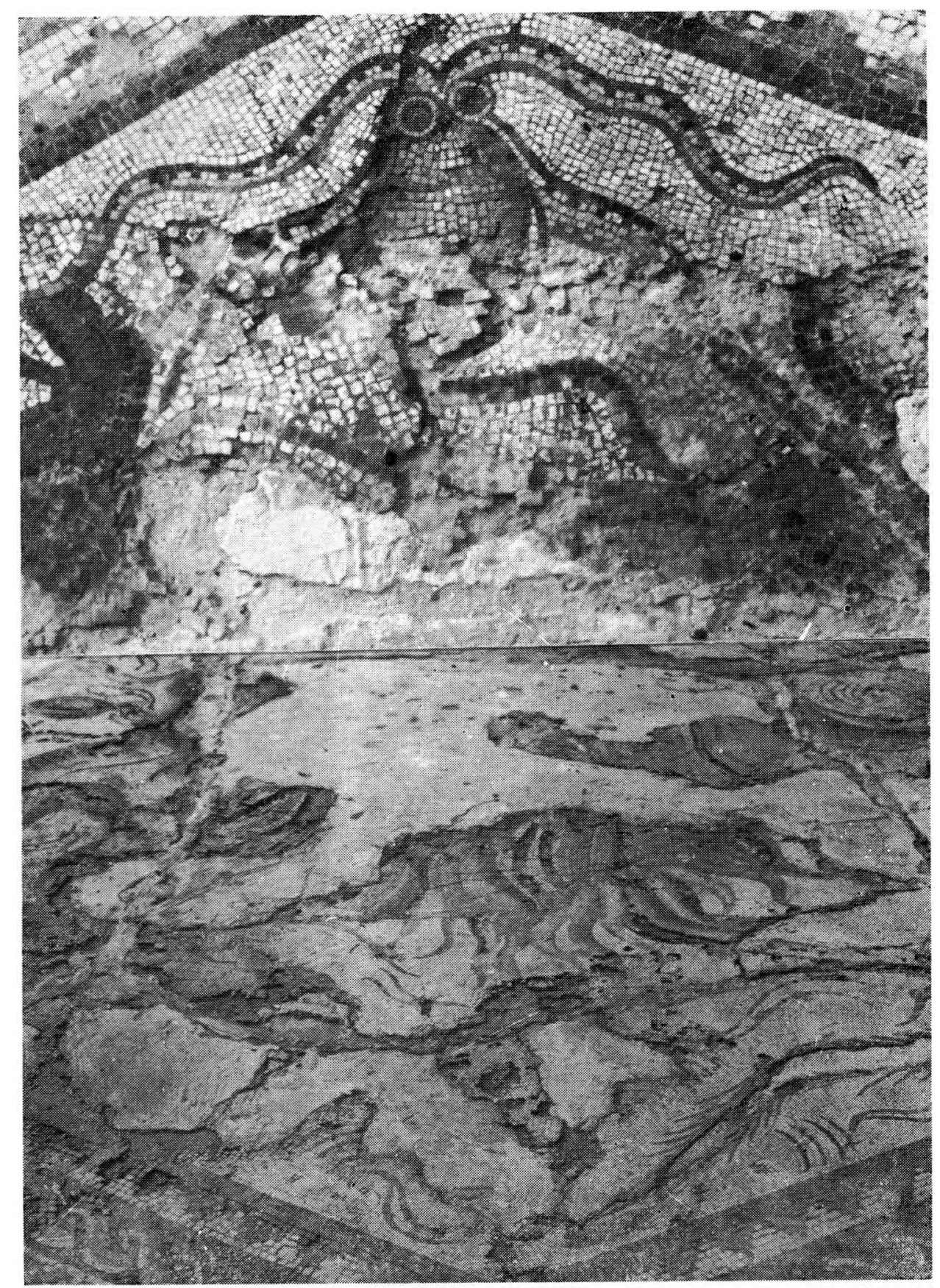

Fig. 9 - Mosaïque d'Océan de Quintanilla de la Cueza, n.o 2 (cliché de l'A.) 


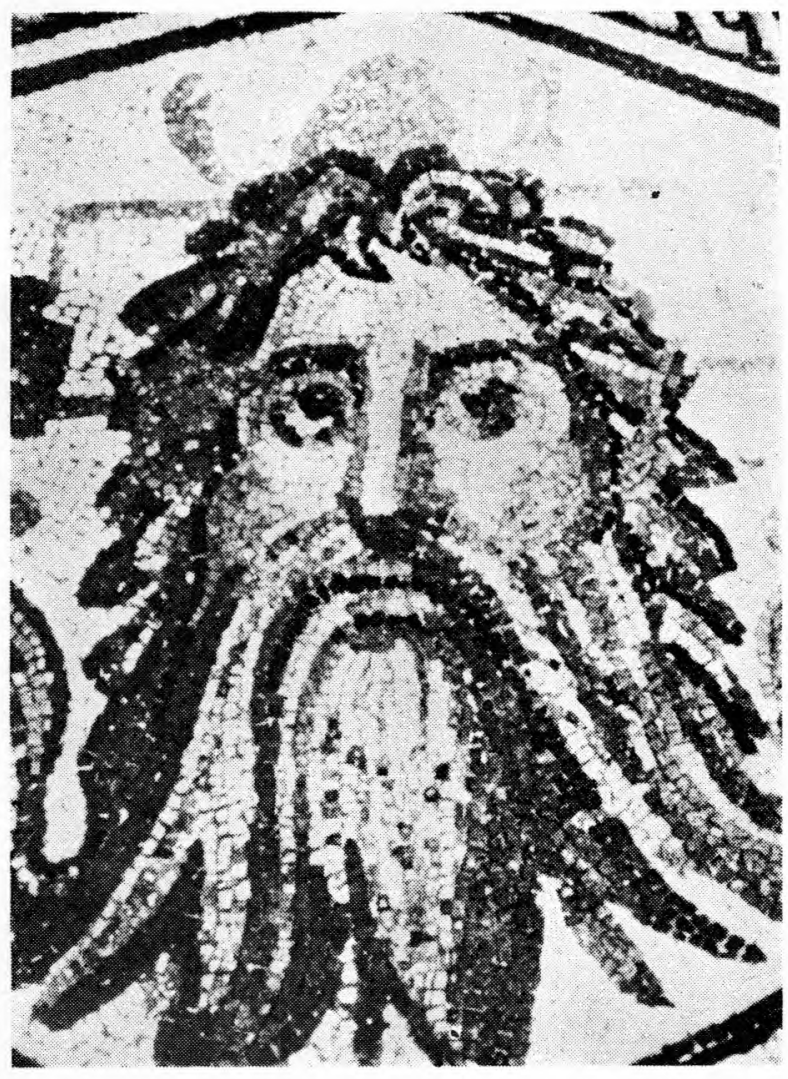

Fig. 10 - Mosaïque d'Océan découverte à Cordoue en 1980 (d'après A. M. Vicent, Arqueologia, 81, p. 152) 


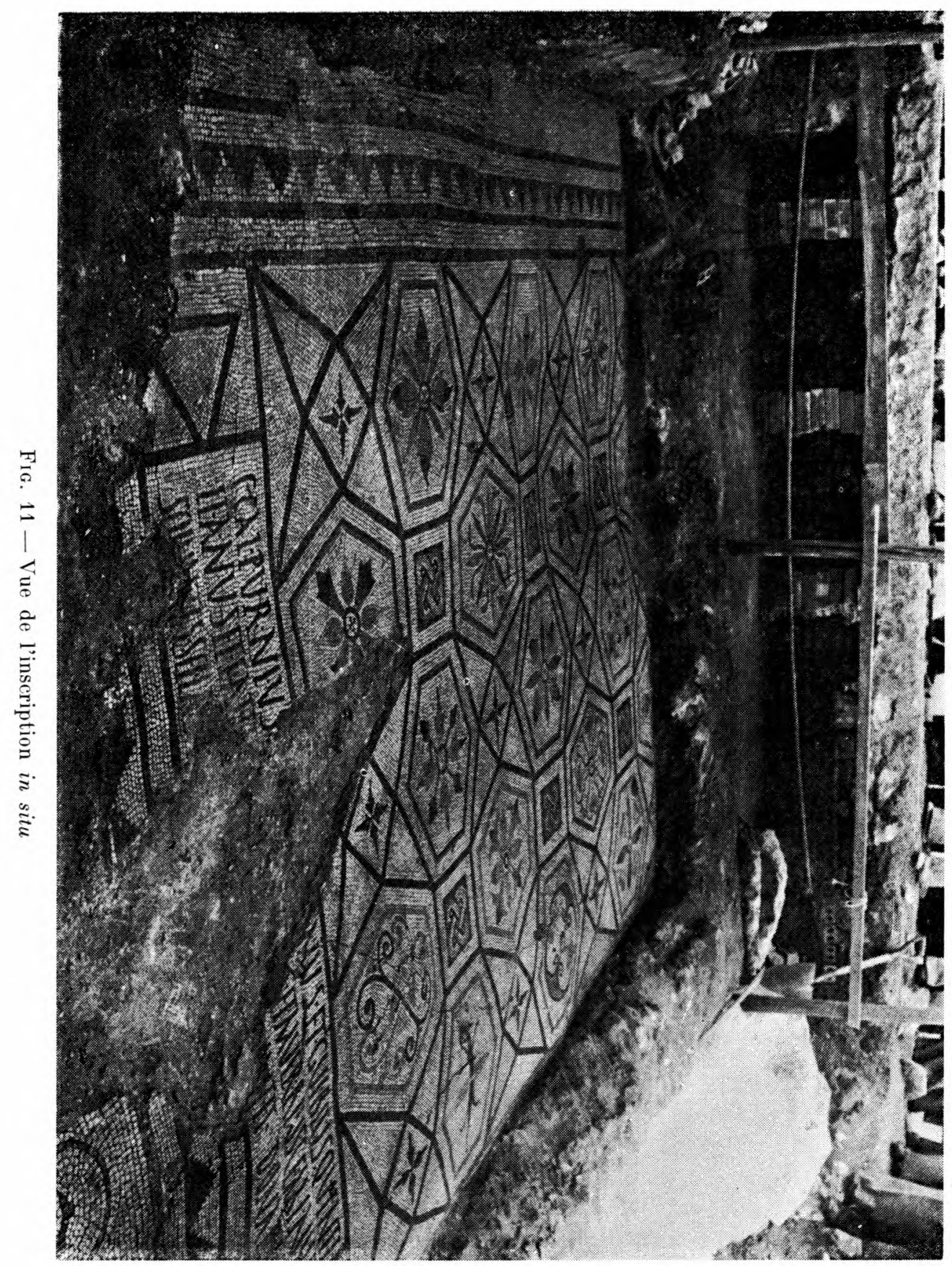




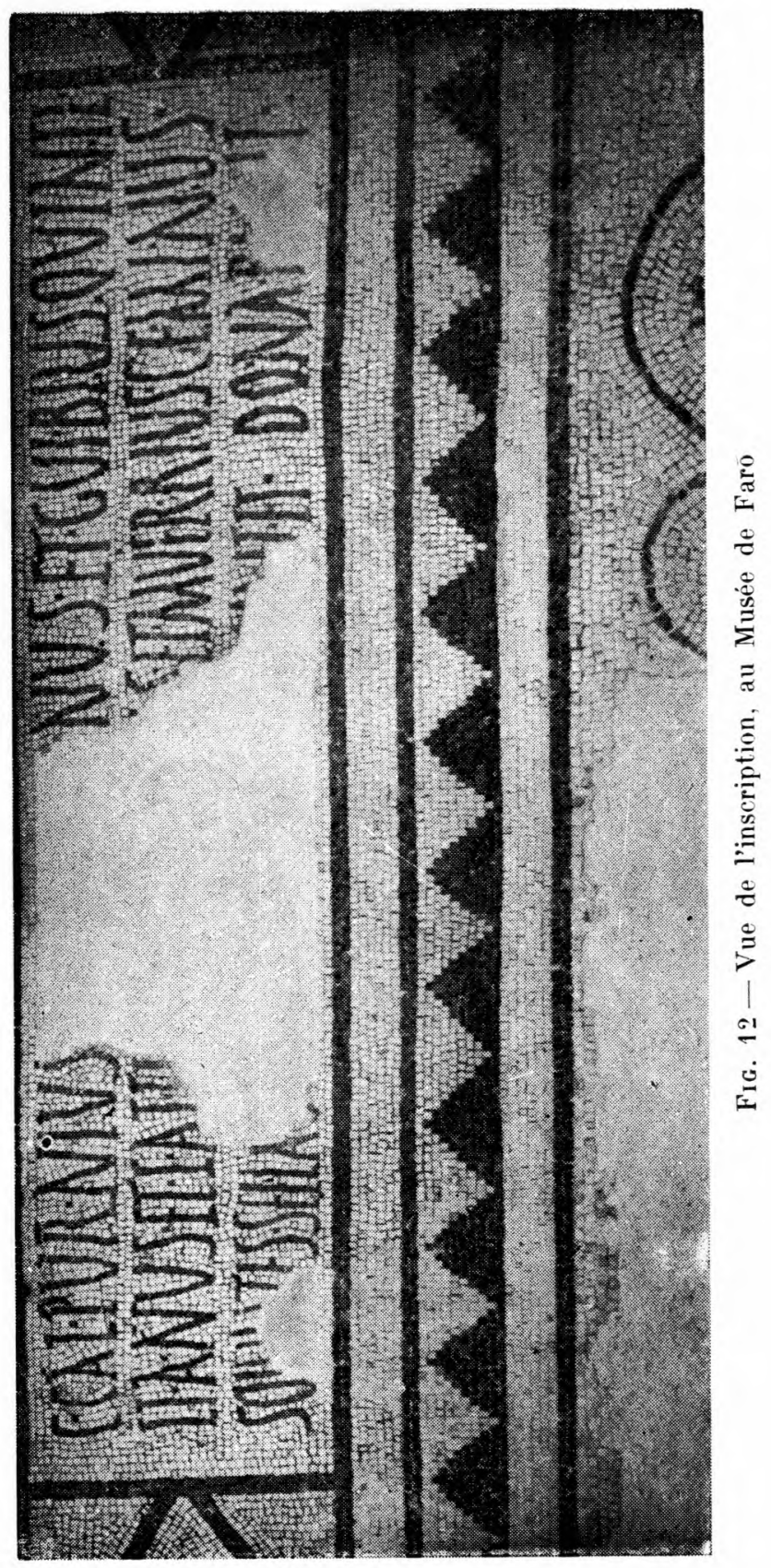

